\title{
Supplement of The SPARC water vapor sssessment II: assessment of satellite measurements of upper tropospheric water vapor
}

William Read ${ }^{1}$, Gabriele Stiller ${ }^{2}$, Stefan Lossow ${ }^{2}$, Michael Kiefer ${ }^{2}$, Farahnaz Khosrawi ${ }^{2}$, Dale Hurst ${ }^{3}$, Holger Vömel ${ }^{4}$, Karen Rosenlof ${ }^{5}$, Bianca M. Dinelli ${ }^{6}$, Piera Raspollini ${ }^{7}$, Gerald E. Nedoluha ${ }^{8}$, John C. Gille ${ }^{9,10}$, Yasuko Kasai ${ }^{11}$, Patrick Eriksson ${ }^{12}$, Christopher E. Sioris ${ }^{13}$, Kaley A. Walker ${ }^{14}$, Katja Weigel $^{15}$, John P. Burrows ${ }^{15}$, and Alexei Rozanov ${ }^{15}$

${ }^{1}$ Jet Propulsion Laboratory, California Institute of Technology, Pasadena, Ca., USA.

${ }^{2}$ Karlsruhe Institute of Technology, Institute of Meteorology and Climate Research, Karlsruhe, Germany.

${ }^{3}$ Global Monitoring Division, NOAA, Earth System Research Laboratory, Boulder, Colorado, USA.

${ }^{4}$ Earth Observing Laboratory, National Center for Atmospheric Research, Boulder, Colorado, USA.

${ }^{5}$ Chemical Science Division, NOAA, Earth System Research Laboratory, Boulder, Colorado, USA.

${ }^{6}$ Instituto di Scienze dell'Atmosfera e del Clima del Consiglio Nazionale delle Ricerche (ISAC-CNR), Via Gobetti, 101, 40129 Bologna, Italy.

${ }^{7}$ Instituto di Fisica Applicata del Consiglio Nazionale delle Ricerche (IFAC-CNR), Via Madonna del Piano, 10, 50019 Sesto Fiorentino, Italy.

${ }^{8}$ Naval Research Laboratory, Remote Sensing Division, 4555 Overlook Avenue Southwest, Washington, DC 20375, USA.

${ }^{9}$ National Center for Atmospheric Research, Atmospheric Chemistry Observations \& Modeling Laboratory, P.O. Box 3000, Boulder, CO. 80307-3000, USA.

${ }^{10}$ University of Colorado, Atmospheric and Oceanic Sciences, Boulder, CO 80309-0311, USA.

${ }^{11}$ National Institute of Information and Communications Technology (NICT), 20 THz Research Center, 4-2-1 Nukui-kita, Koganei, Tokyo 184-8795, Japan.

${ }^{12}$ Chalmers University of Technology, Department of Space, Earth and Environment, Hörsalsvägen 11, 41296 Göteborg, Sweden.

${ }^{13}$ York University, Center for Research in Earth and Space Science, 4700 Keele Street, Toronto, Ontario M3J 1P3, Canada.

${ }^{14}$ University of Toronto, Department of Physics, 60 St. George Street, Toronto, Ontario M5S 1A7, Canada.

${ }^{15}$ University of Bremen, Institute of Environmental Physics, Otto-Hahn-Allee 1, 28334 Breman, Germany.

Correspondence: Read (william.g.read@jpl.nasa.gov)

Copyright statement.

\section{S1 Introduction}

Figures S1-S30 show coincident scatter plots for several instruments versus MLS-Aura or MIPAS-ESA either as a probability density function (PDF, Figure 16 in the main manuscript) or as a scatter of the individual coincidences when their number are few. Figures S31-S33 Show gridded mapped comparisons for other heights in the same format as Figure 21 in the main manuscript. Figures S34-S36 Show a scatter plot of the grid box averages for other heights in the same format as Figure 22 in the main manuscript. Figures S37-S39 Show mapped humidity fields for the SMILES products for several heights in the 
same format as Figure 21 in the main manuscript. Figures S40-S42 Show a scatter plot of the grid box averages for SMILES humidity product versus MLS-Aura for several heights in the same format as Figure 22 in the main manuscript. Figures S4310 S44 Show mapped humidity fields for the occultation instruments for several heights in the same format as Figure 21 in the main manuscript. Figures S45-S46 Show a scatter plot of the grid box averages for the occultation instruments versus MLS-Aura for several heights in the same format as Figure 22 in the main manuscript. 


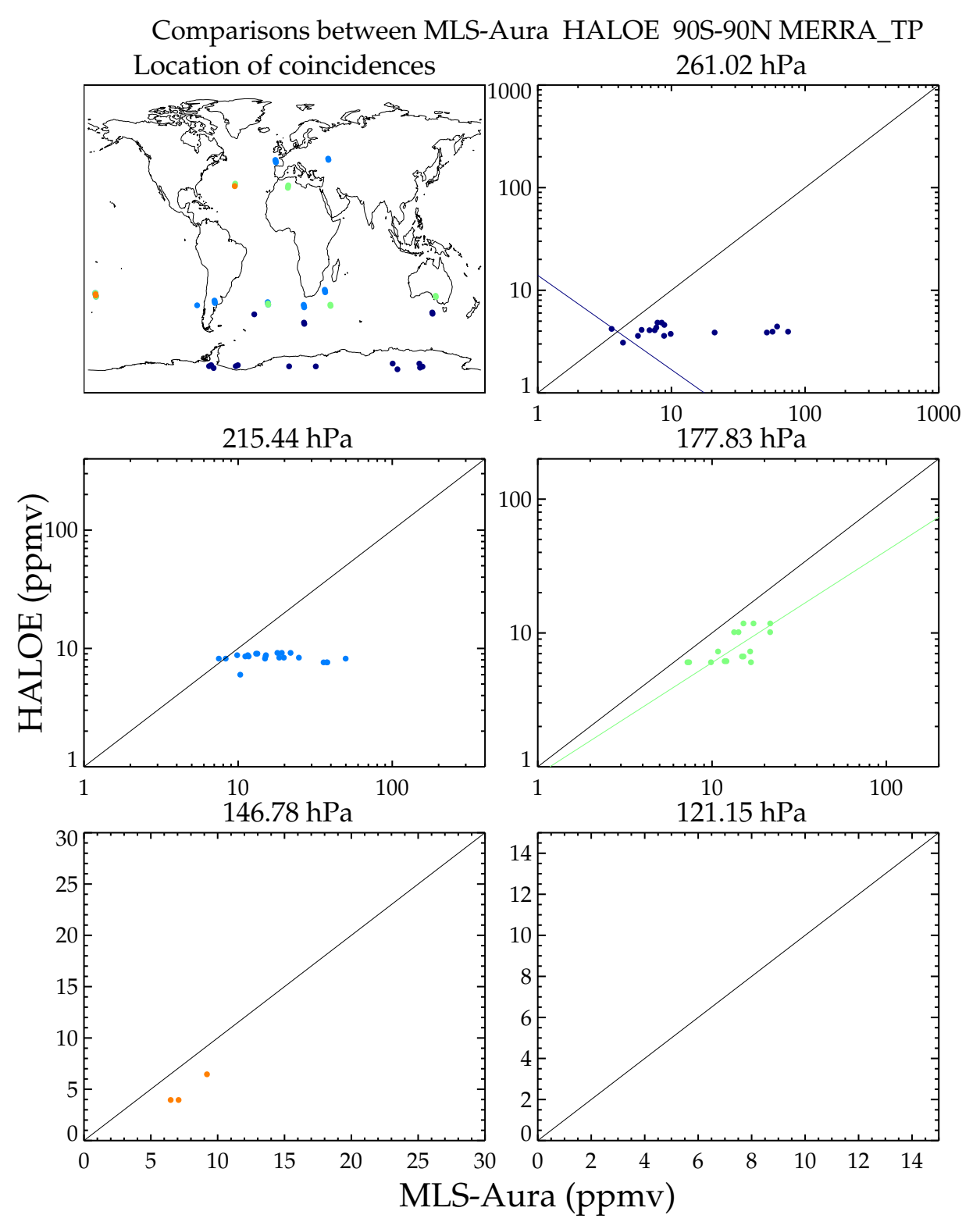

Figure S1. Scatter plot of coincident humidity measurements between MLS-Aura and HALOE 


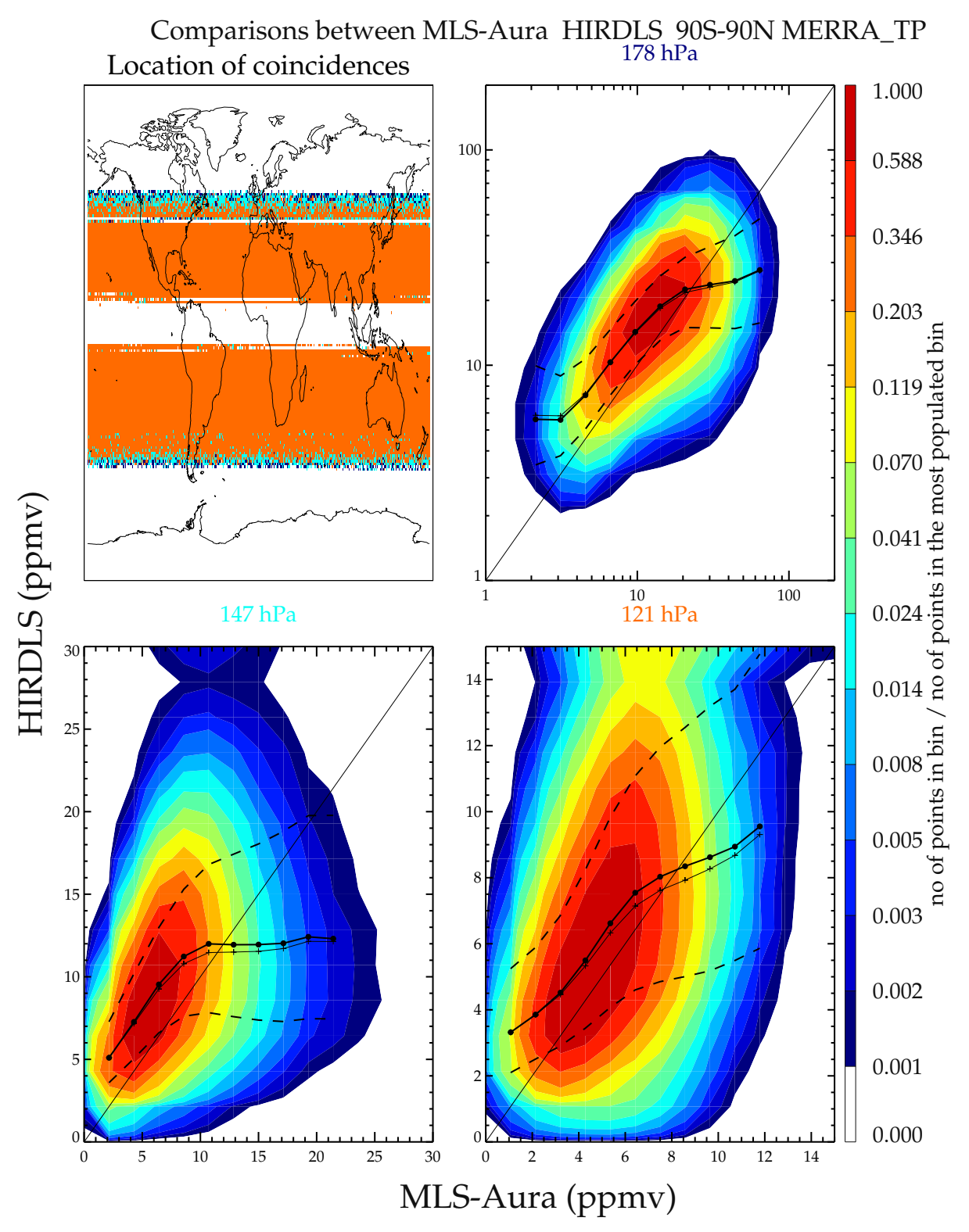

Figure S2. Scatter plot of coincident humidity measurements between MLS-Aura and HIRDLS 
Comparisons between MLS-Aura MIPAS-Bologna 90S-90N MERRA_TP Location of coincidences
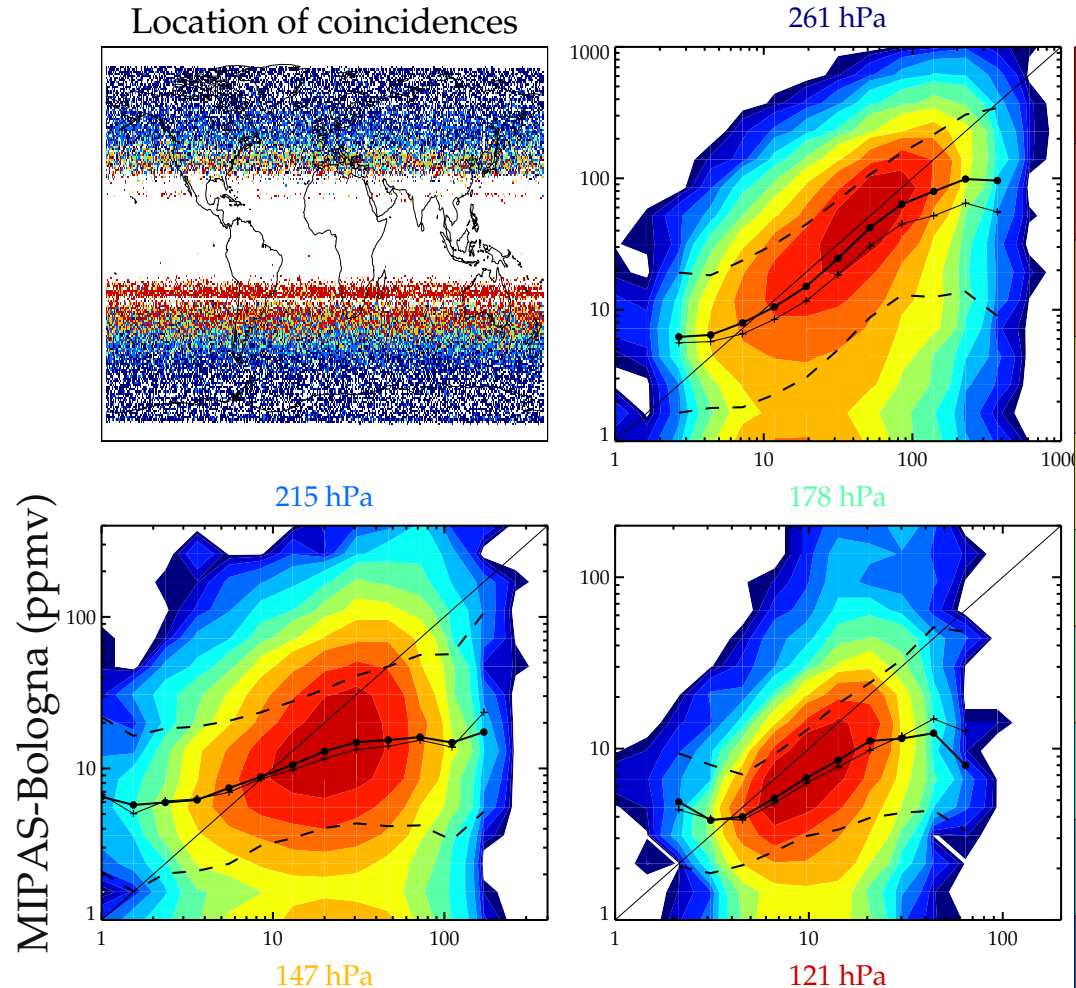

1.000

0.588

0.346

0.203

0.119

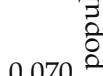

$0.070 \frac{2}{0}$

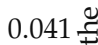

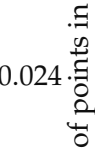

$0.014 \stackrel{?}{\varrho}$

0.008

$147 \mathrm{hPa}$

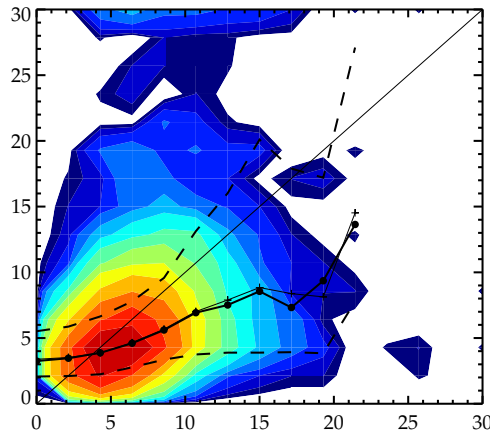

$121 \mathrm{hPa}$

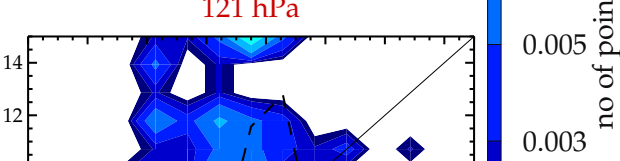

MLS-Aura (ppmv)

Figure S3. Scatter plot of coincident humidity measurements between MLS-Aura and MIPAS-Bologna 
Comparisons between MLS-Aura MIPAS-ESA 90S-90N MERRA_TP Location of coincidences
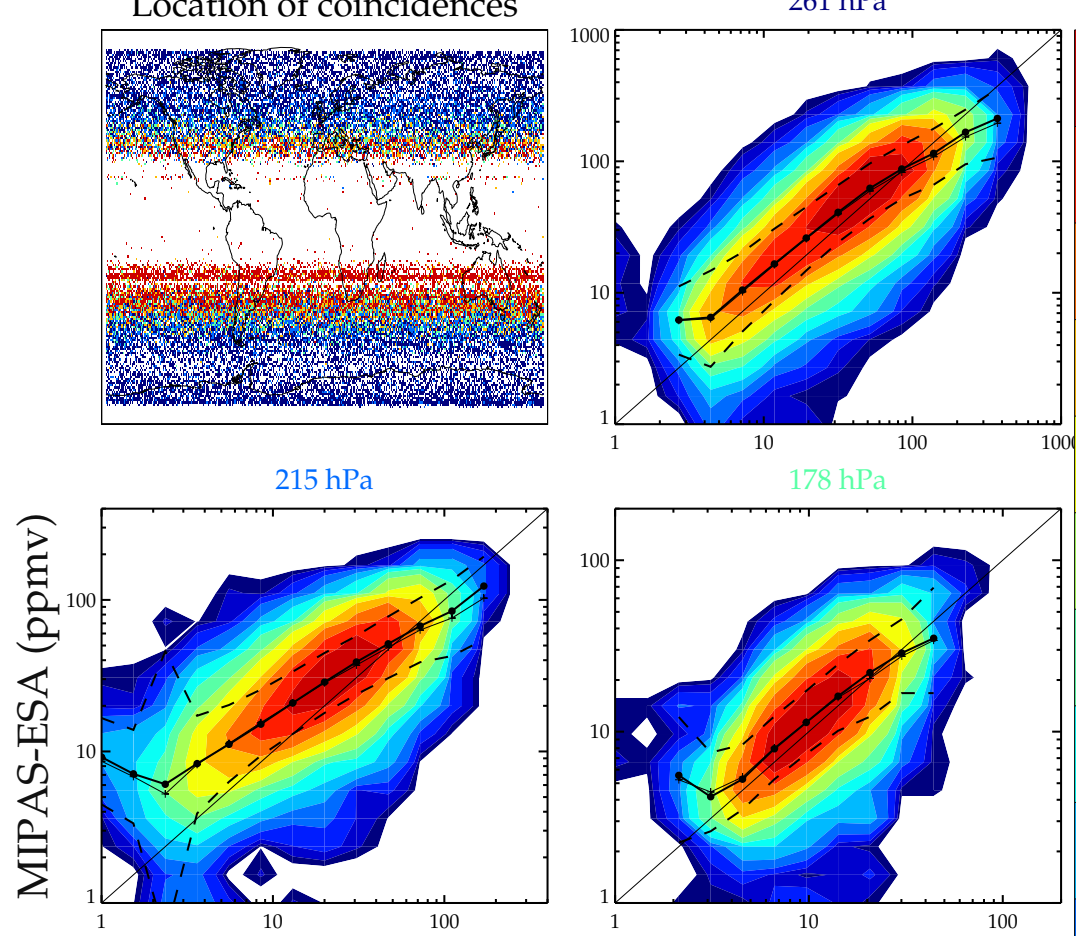

1.000

0.588

0.346

0.203

0.119

薃

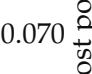

0.041

0.024

状

$0.014 \stackrel{?}{\Xi}$

0.008 ق

$147 \mathrm{hPa}$

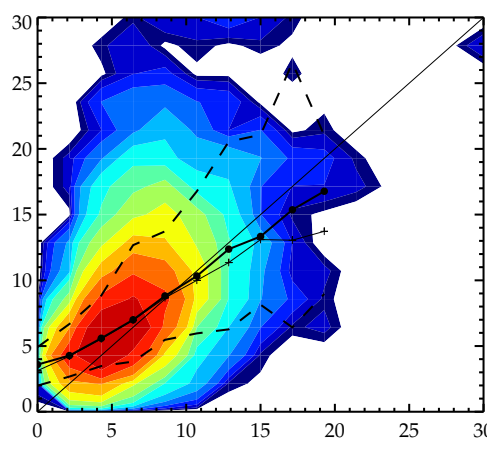

$121 \mathrm{hPa}$

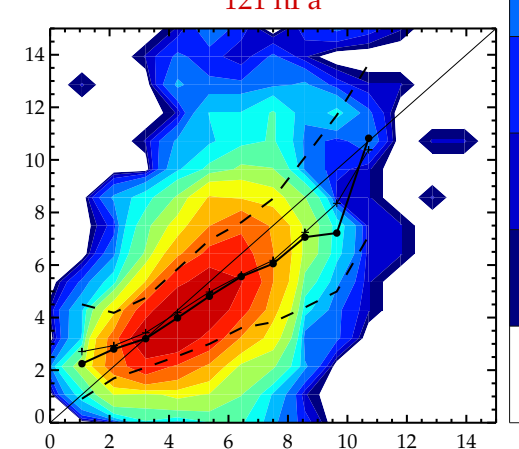

$0.005 \stackrel{0}{\circ}$

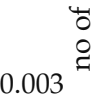

0.002

0.001

0.000

MLS-Aura (ppmv)

Figure S4. Scatter plot of coincident humidity measurements between MLS-Aura and MIPAS-ESA 
Comparisons between MLS-Aura MIPAS-Oxford 90S-90N MERRA_TP Location of coincidences
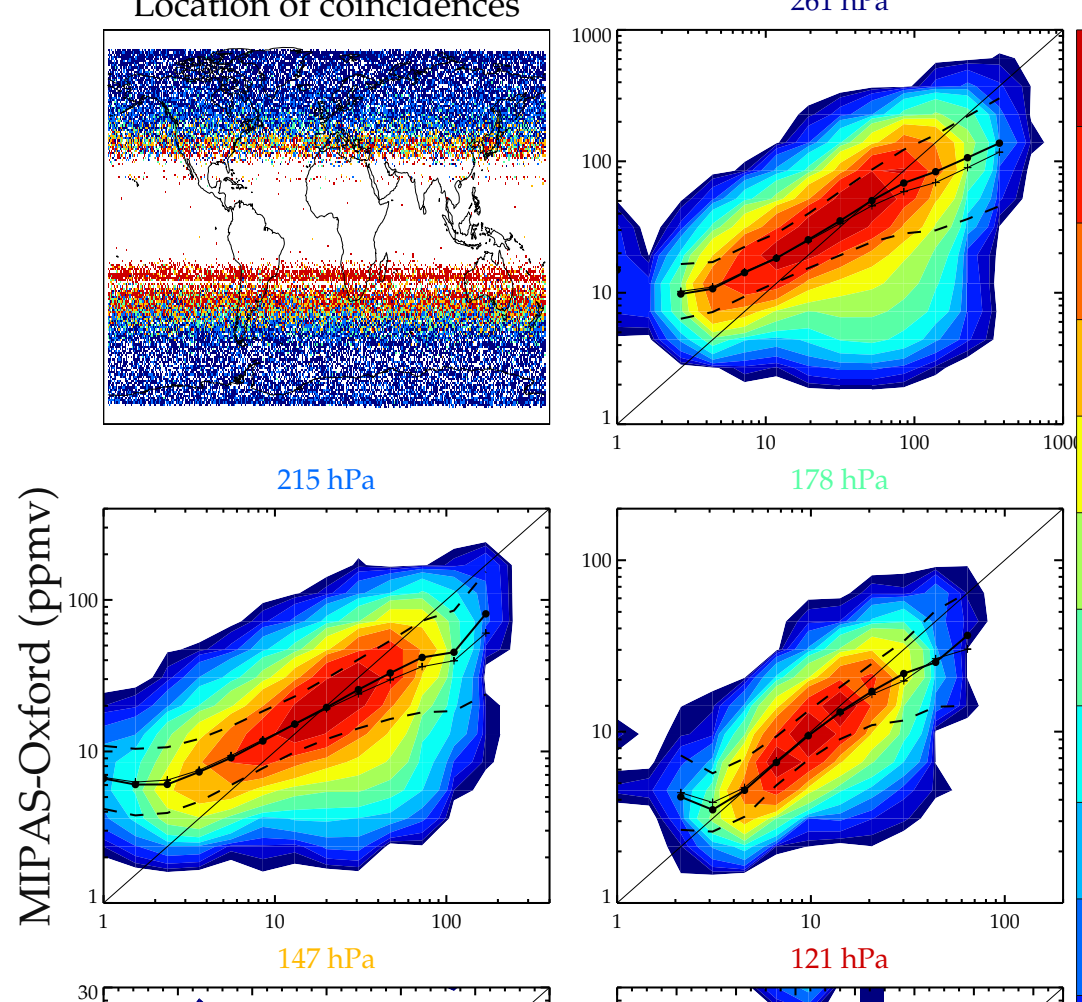

1.000

0.588

0.346

0.203

0.119

(ำ

0.070 色

$0.041 \stackrel{乛}{\frac{1}{9}}$

0.024 .

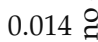

0.008

$121 \mathrm{hPa}$
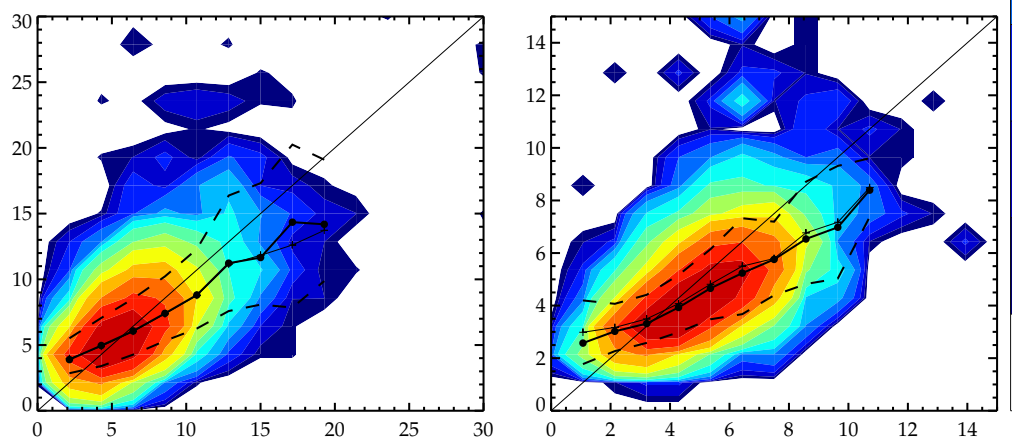

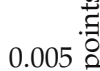

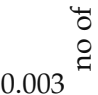

0.002

0.001

MLS-Aura (ppmv)

Figure S5. Scatter plot of coincident humidity measurements between MLS-Aura and MIPAS-Oxford 


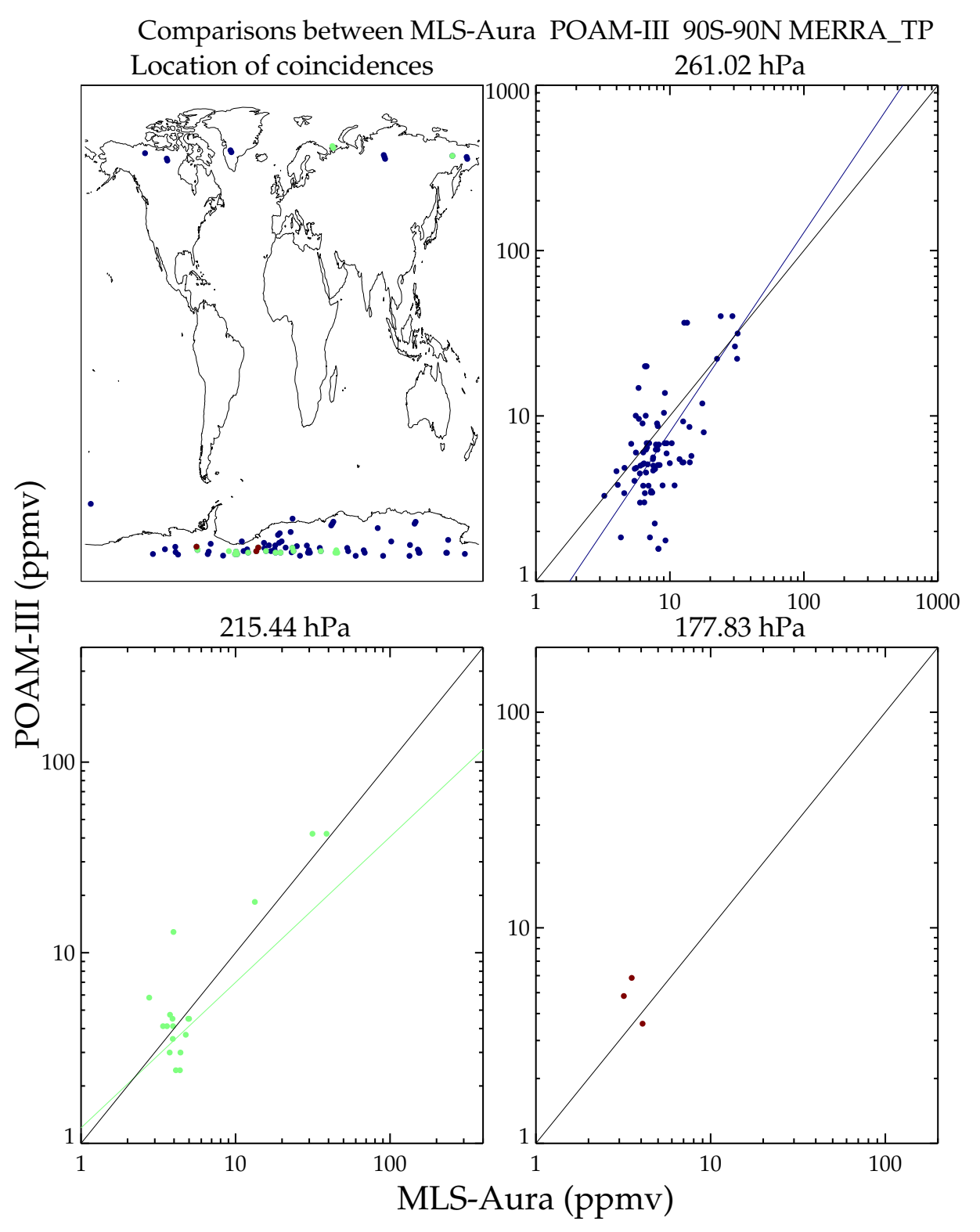

Figure S6. Scatter plot of coincident humidity measurements between MLS-Aura and POAM-III 
Comparisons between MLS-Aura SAGE-II 90S-90N MERRA_TP Location of coincidences
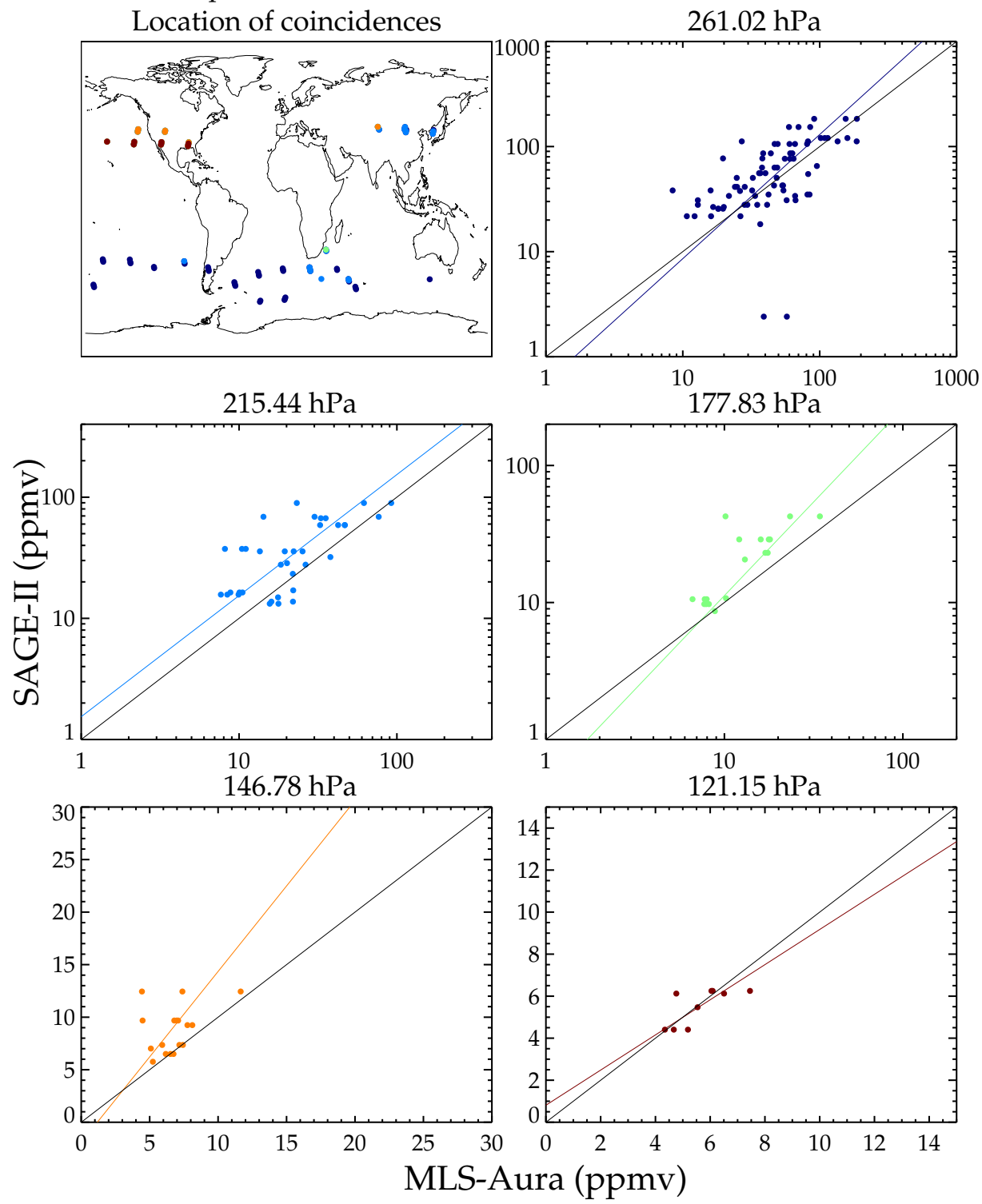

Figure S7. Scatter plot of coincident humidity measurements between MLS-Aura and SAGE-II 


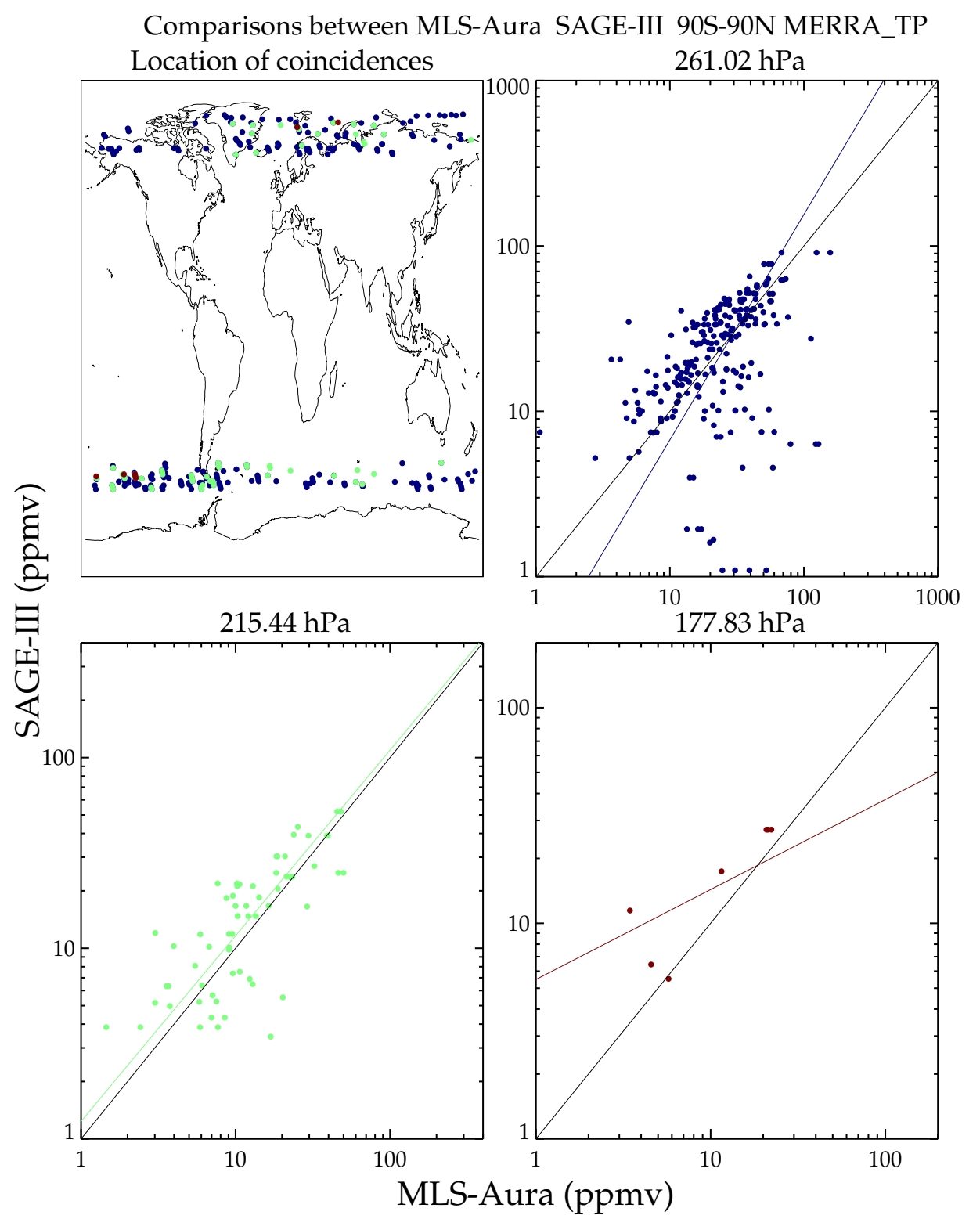

Figure S8. Scatter plot of coincident humidity measurements between MLS-Aura and SAGE-III 
Comparisons between MLS-Aura SCIAMACHY 90S-90N MERRA_TP Location of coincidences
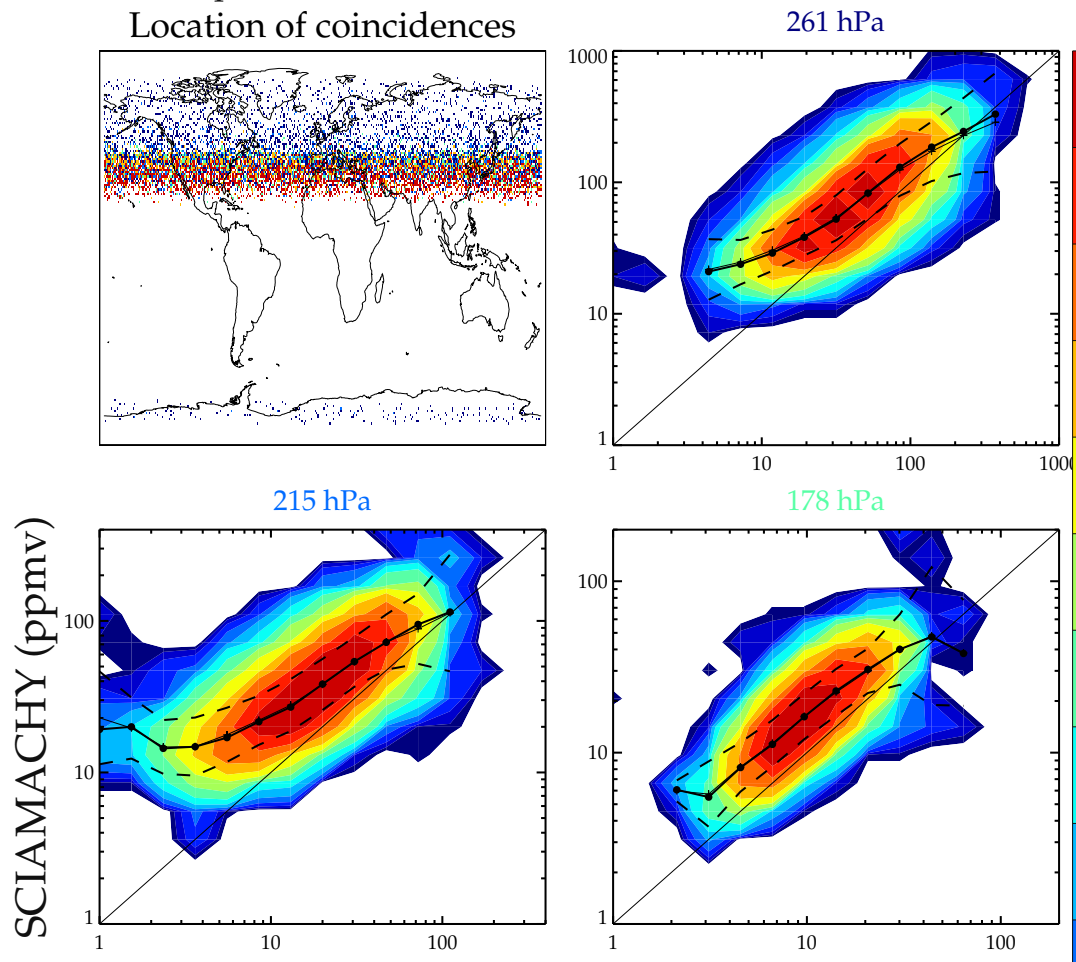

$147 \mathrm{hPa}$
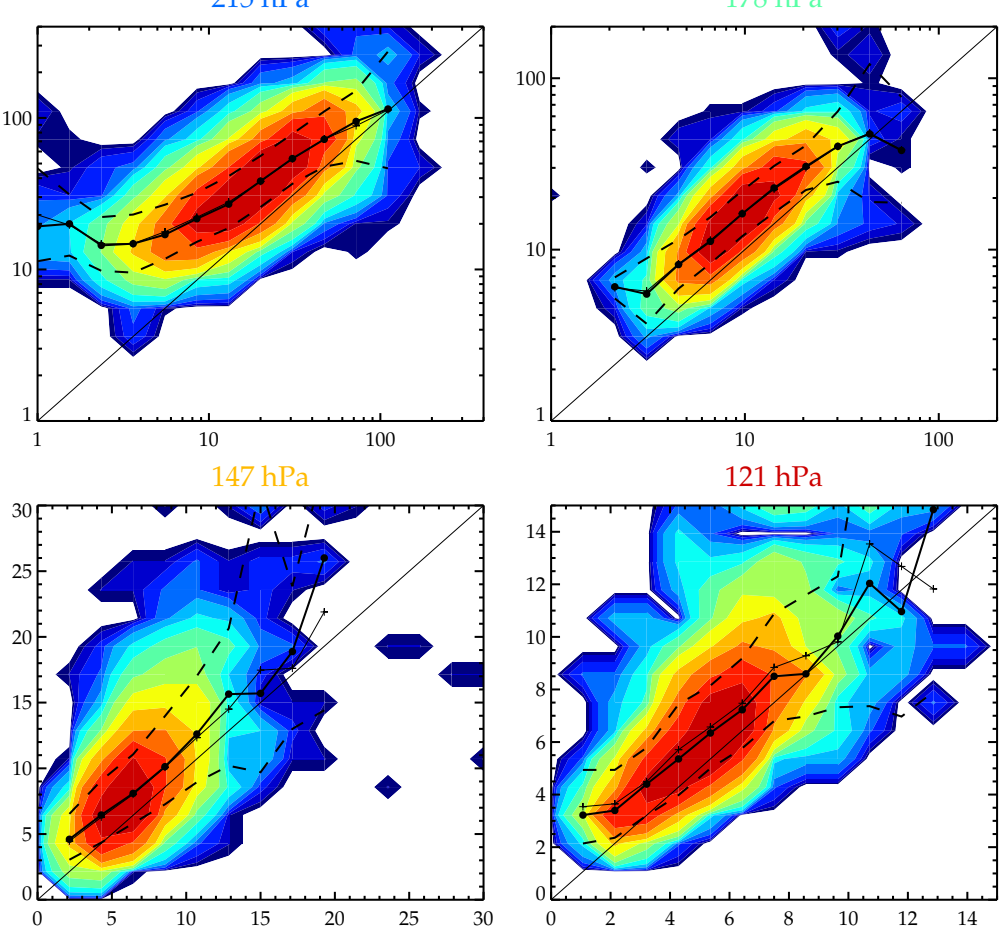

1.000

0.588

0.346

0.203

0.119

है

$0.070 \frac{2}{\frac{0}{0}}$

0.041

0.024 .

$0.014 \stackrel{\varrho}{\complement}$

0.008

$121 \mathrm{hPa}$

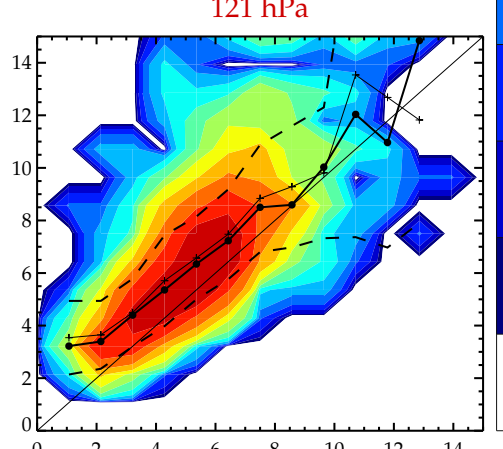

0.005 苞

0.003 ?

0.002

0.001

MLS-Aura (ppmv)

Figure S9. Scatter plot of coincident humidity measurements between MLS-Aura and SCIAMACHY 
Comparisons between MLS-Aura SMILES-Chalmers-AB 90S-90N MERRA_TP Location of coincidences

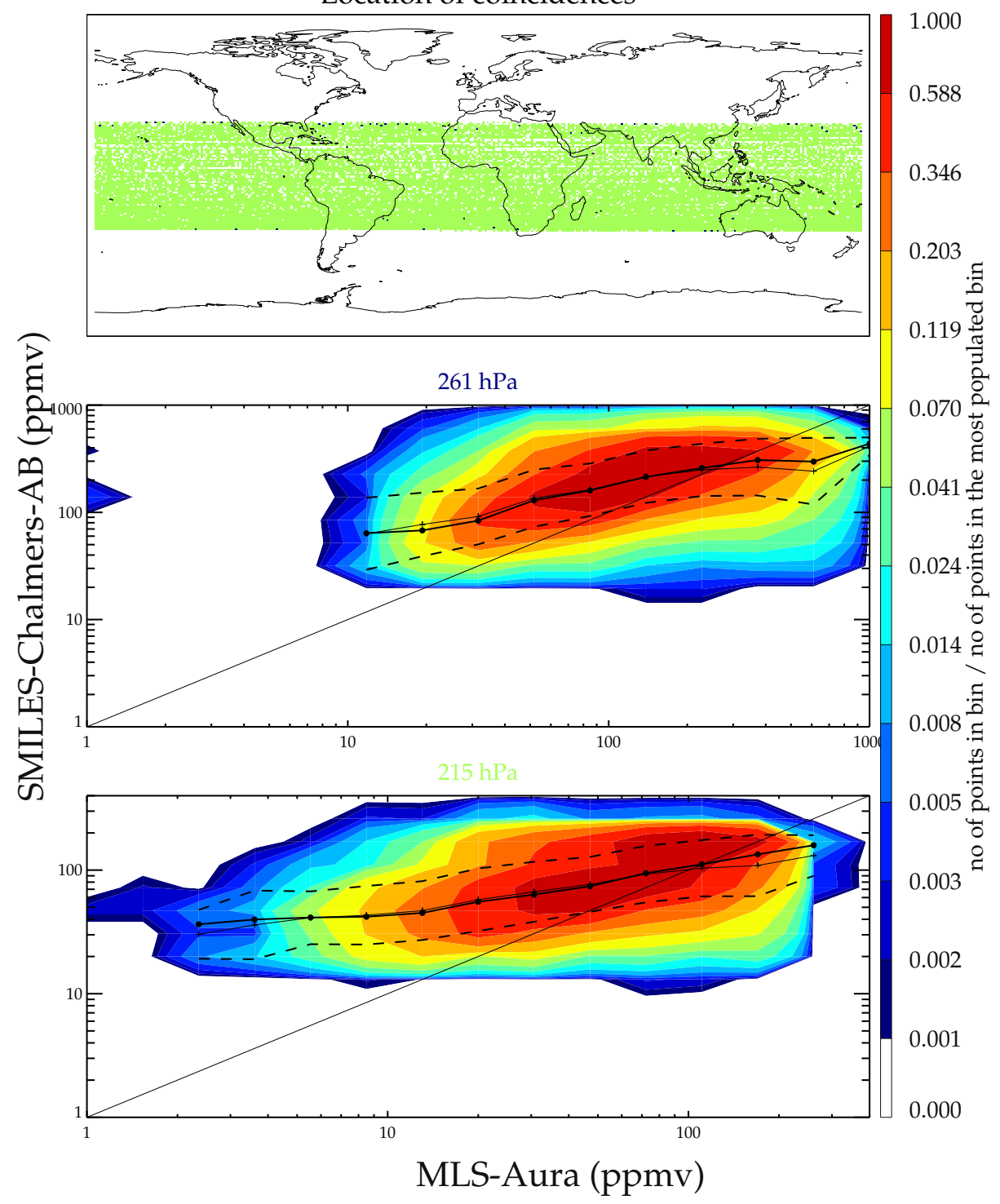

Figure S10. Scatter plot of coincident humidity measurements between MLS-Aura and SMILES Chalmers AB 
Comparisons between MLS-Aura SMILES-Chalmers-CA 90S-90N MERRA_TP Location of coincidences

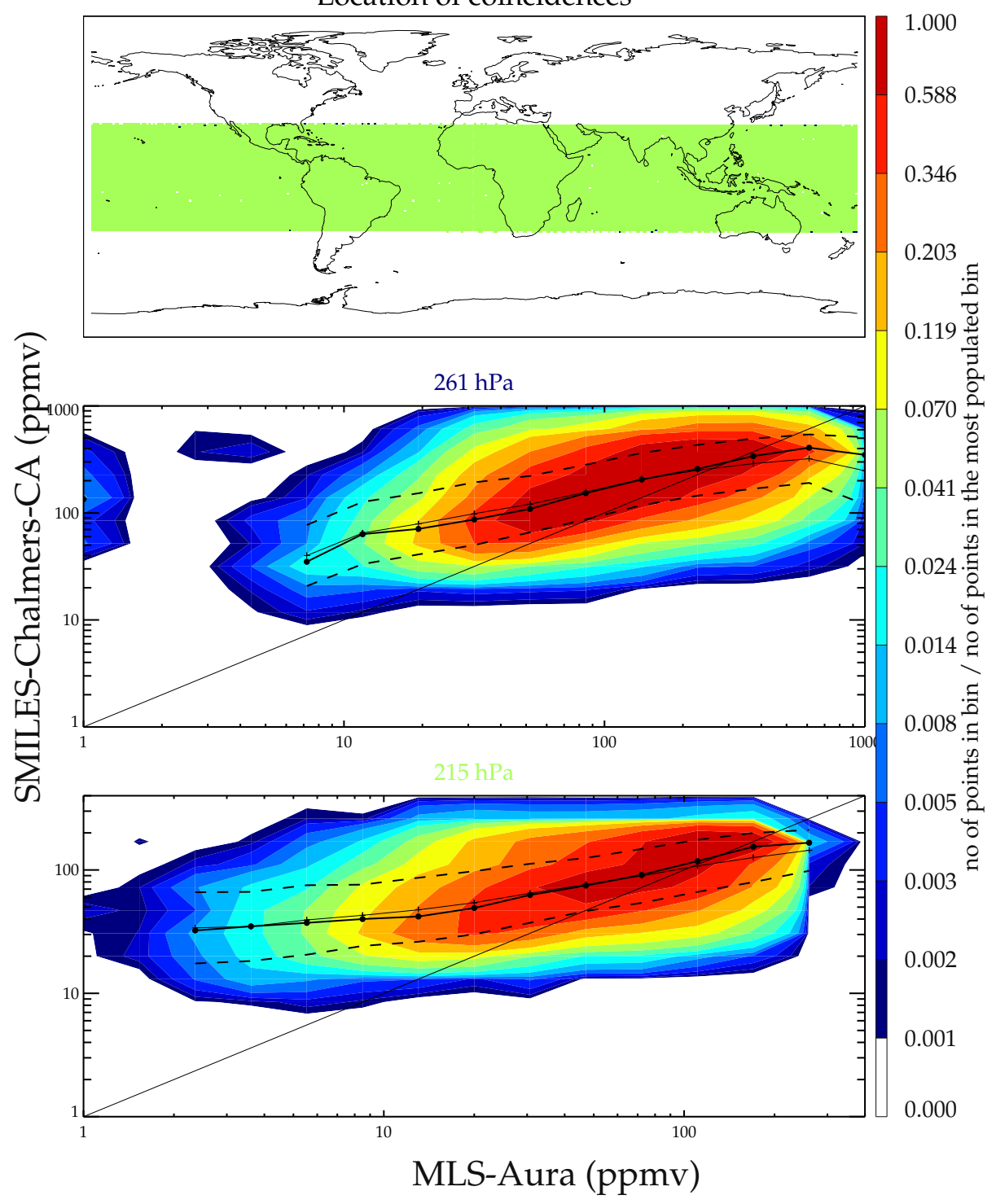

Figure S11. Scatter plot of coincident humidity measurements between MLS-Aura and SMILES Chalmers CA 
Comparisons between MLS-Aura SMILES-Chalmers-CB 90S-90N MERRA_TP Location of coincidences

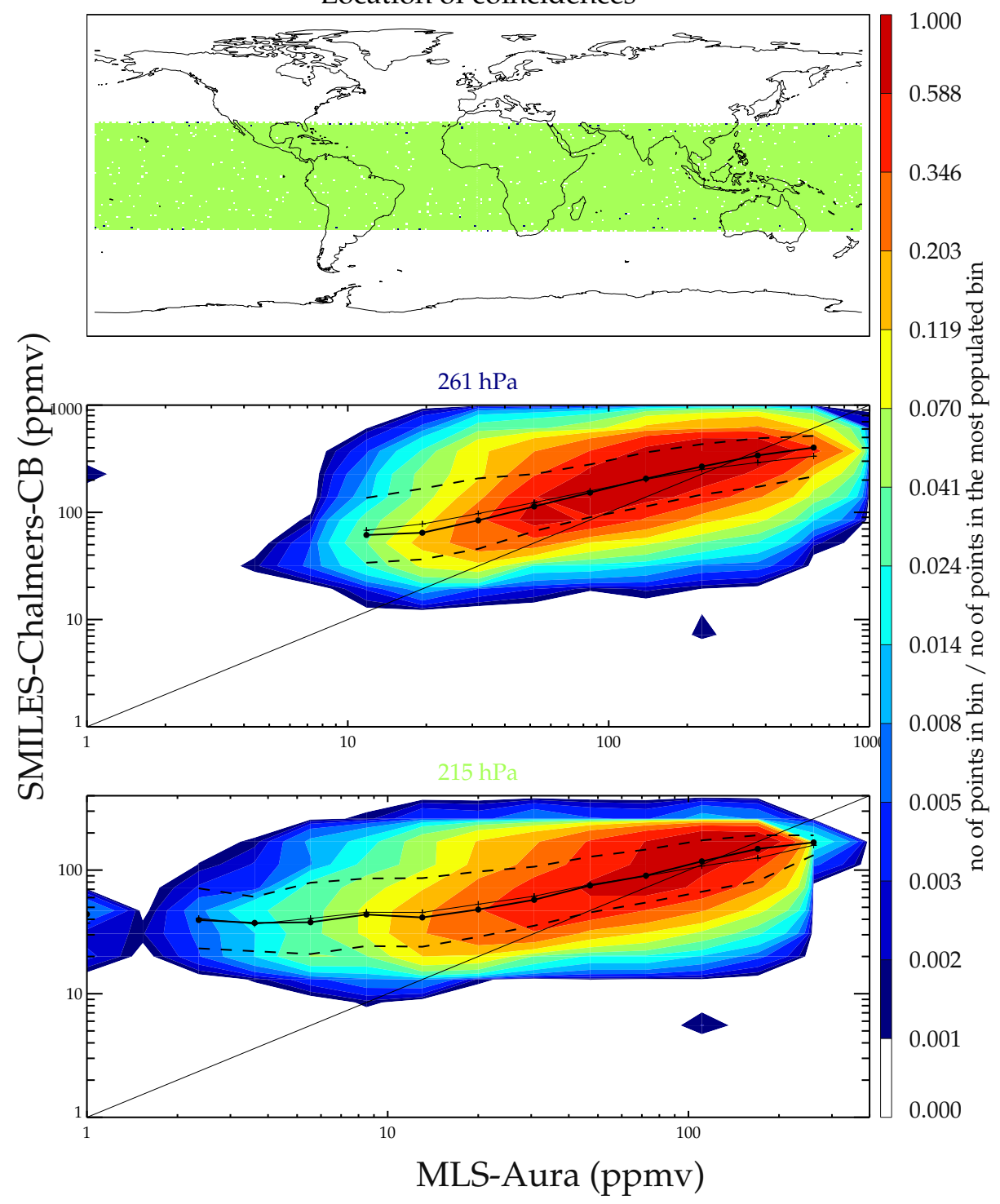

Figure S12. Scatter plot of coincident humidity measurements between MLS-Aura and SMILES Chalmers CB 
Comparisons between MLS-Aura SMILES-JPL-A 90S-90N MERRA_TP Location of coincidences
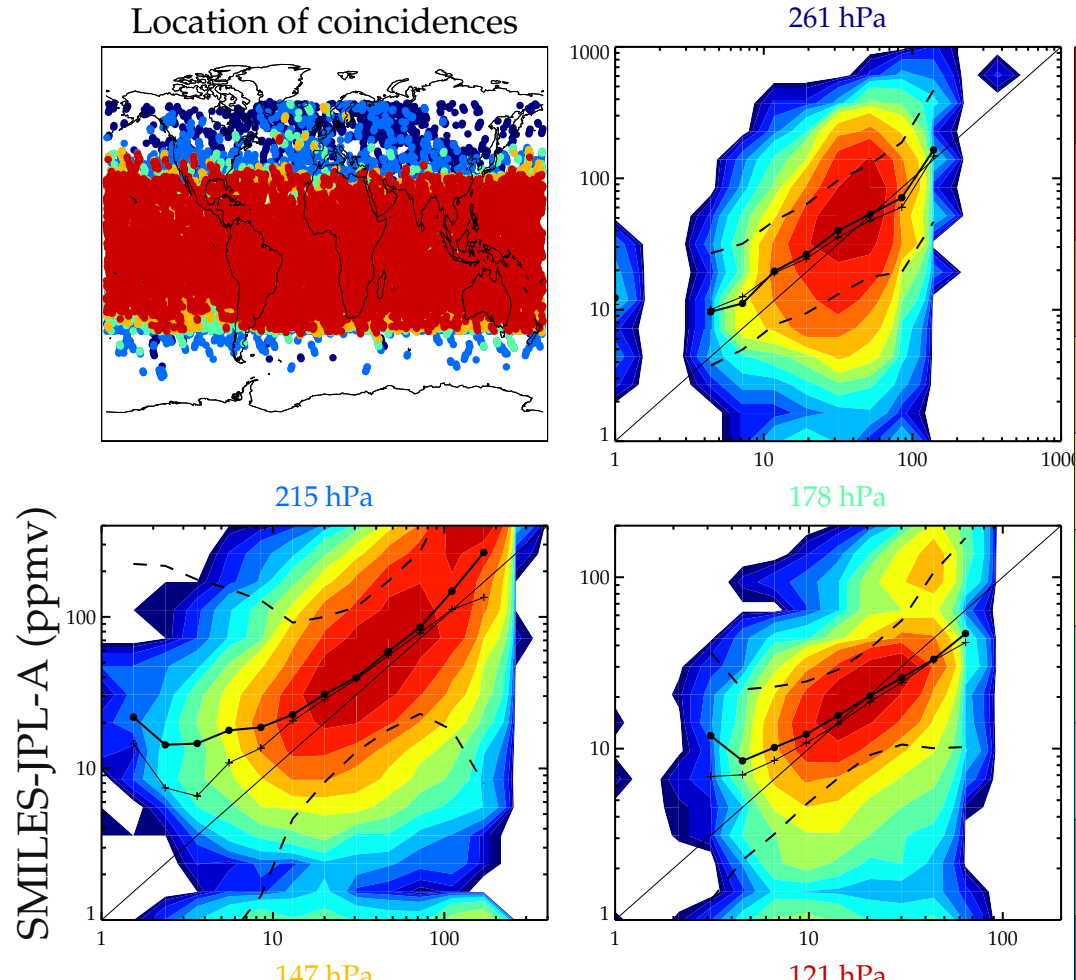

1.000

0.588

0.346

0.203

0.119

$\frac{2}{2}$

$0.070 \stackrel{\frac{1}{0}}{0}$

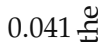

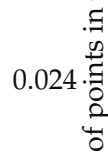

$0.014 \stackrel{ }{\varrho}$

$0.008 \stackrel{\Xi}{\Xi}$

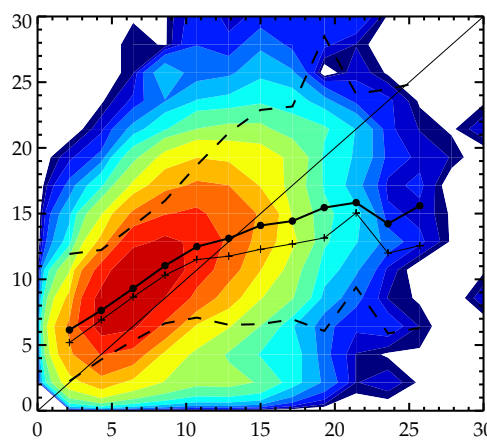

$121 \mathrm{hPa}$
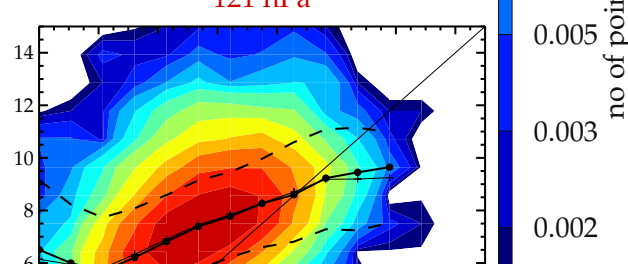

0.002

0.001

MLS-Aura (ppmv)

Figure S13. Scatter plot of coincident humidity measurements between MLS-Aura and SMILES JPL A 
Comparisons between MLS-Aura SMILES-JPL-B 90S-90N MERRA_TP Location of coincidences
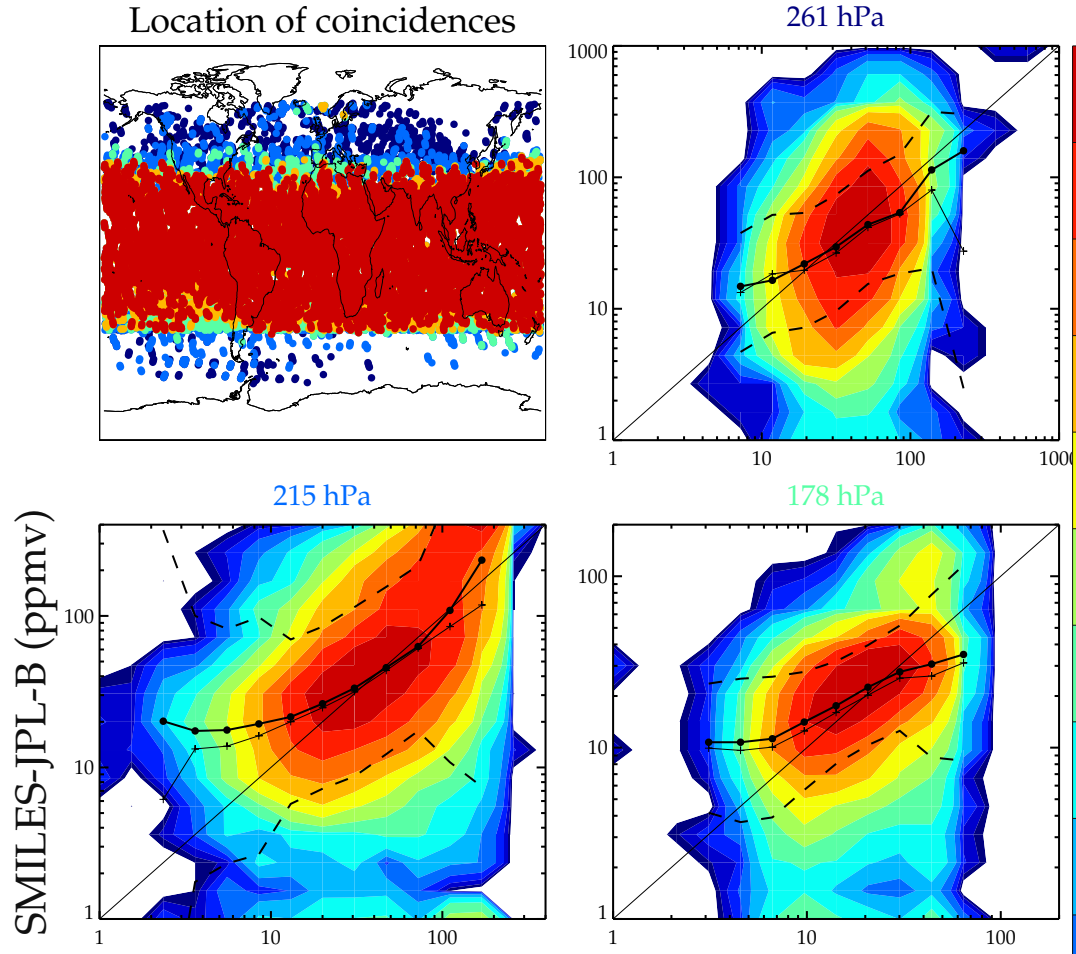

1.000

0.588

0.346

0.203

$0.119 \frac{8}{8}$

范

$147 \mathrm{hPa}$

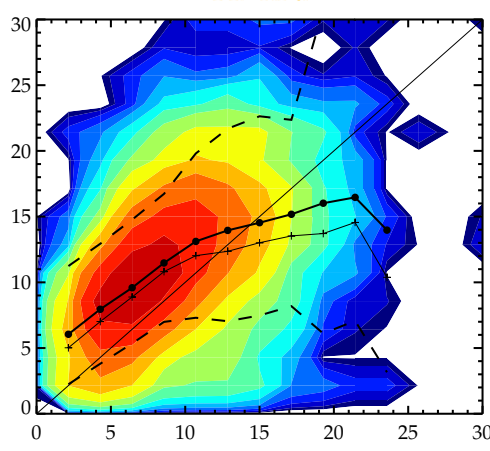

$121 \mathrm{hPa}$

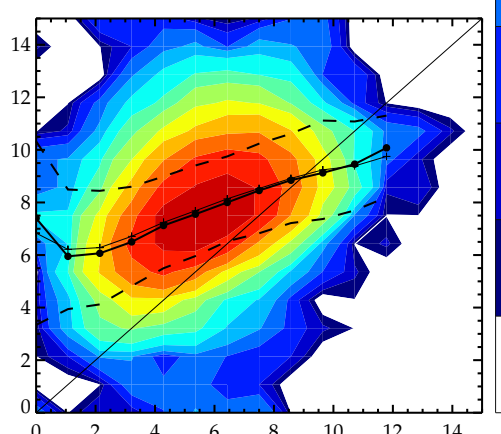
है

$0.041 \stackrel{?}{\oplus}$

0.024 .

$0.014 \stackrel{?}{\jmath}$

$0.008 \stackrel{\Xi}{\Xi}$

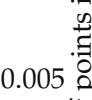

$0.003 \stackrel{8}{?}$

0.002

0.001

MLS-Aura (ppmv)

Figure S14. Scatter plot of coincident humidity measurements between MLS-Aura and SMILES JPL B 
Comparisons between MLS-Aura SMILES-JPL-C 90S-90N MERRA_TP Location of coincidences
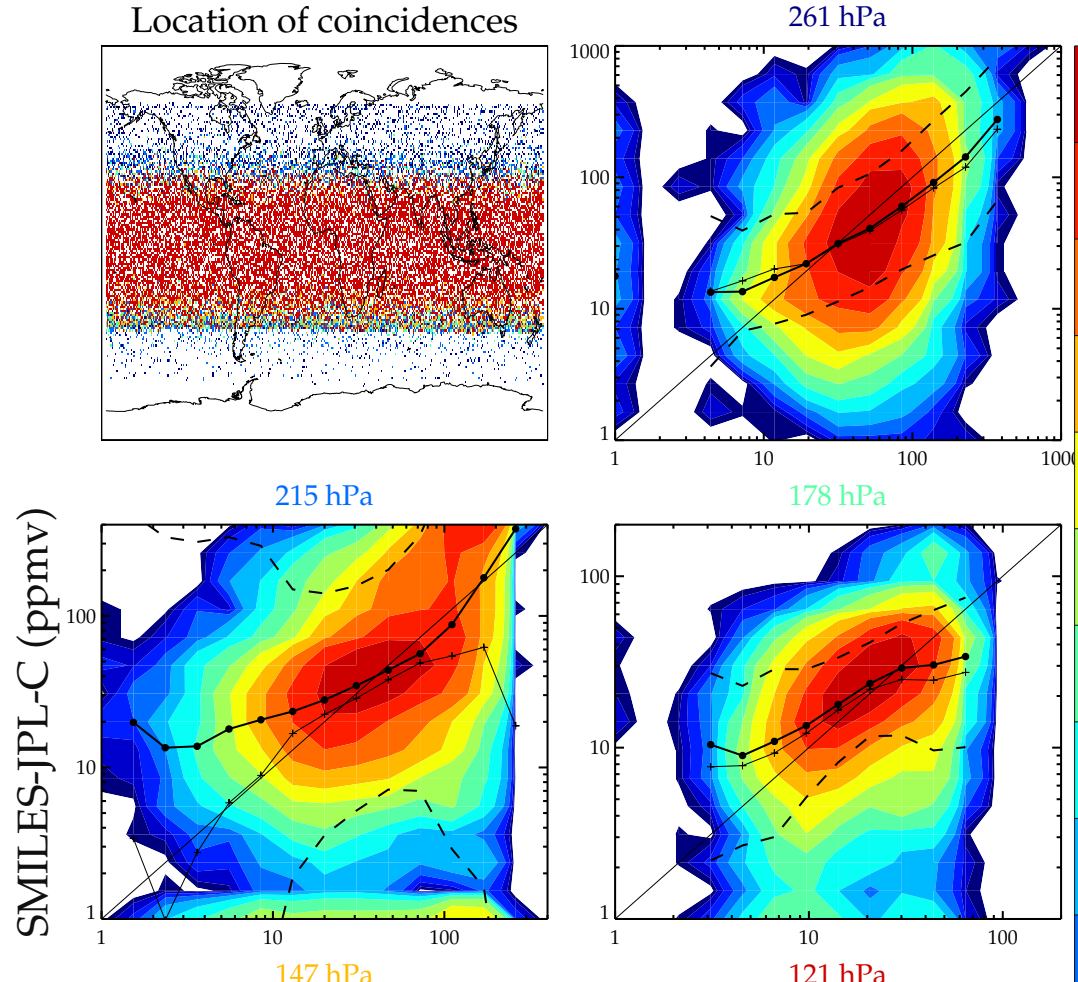

1.000

0.588

0.346

0.203

0.119

है

$0.070 \frac{2}{\frac{0}{0}}$

$0.041 \stackrel{乛}{\simeq}$

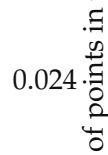

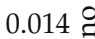

0.008

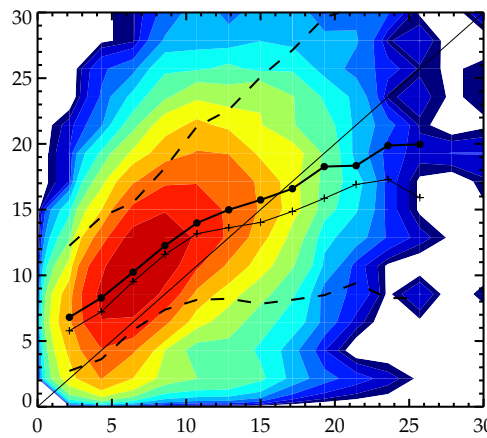

$121 \mathrm{hPa}$

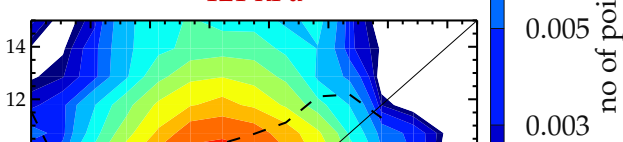

MLS-Aura (ppmv)

Figure S15. Scatter plot of coincident humidity measurements between MLS-Aura and SMILES JPL C 


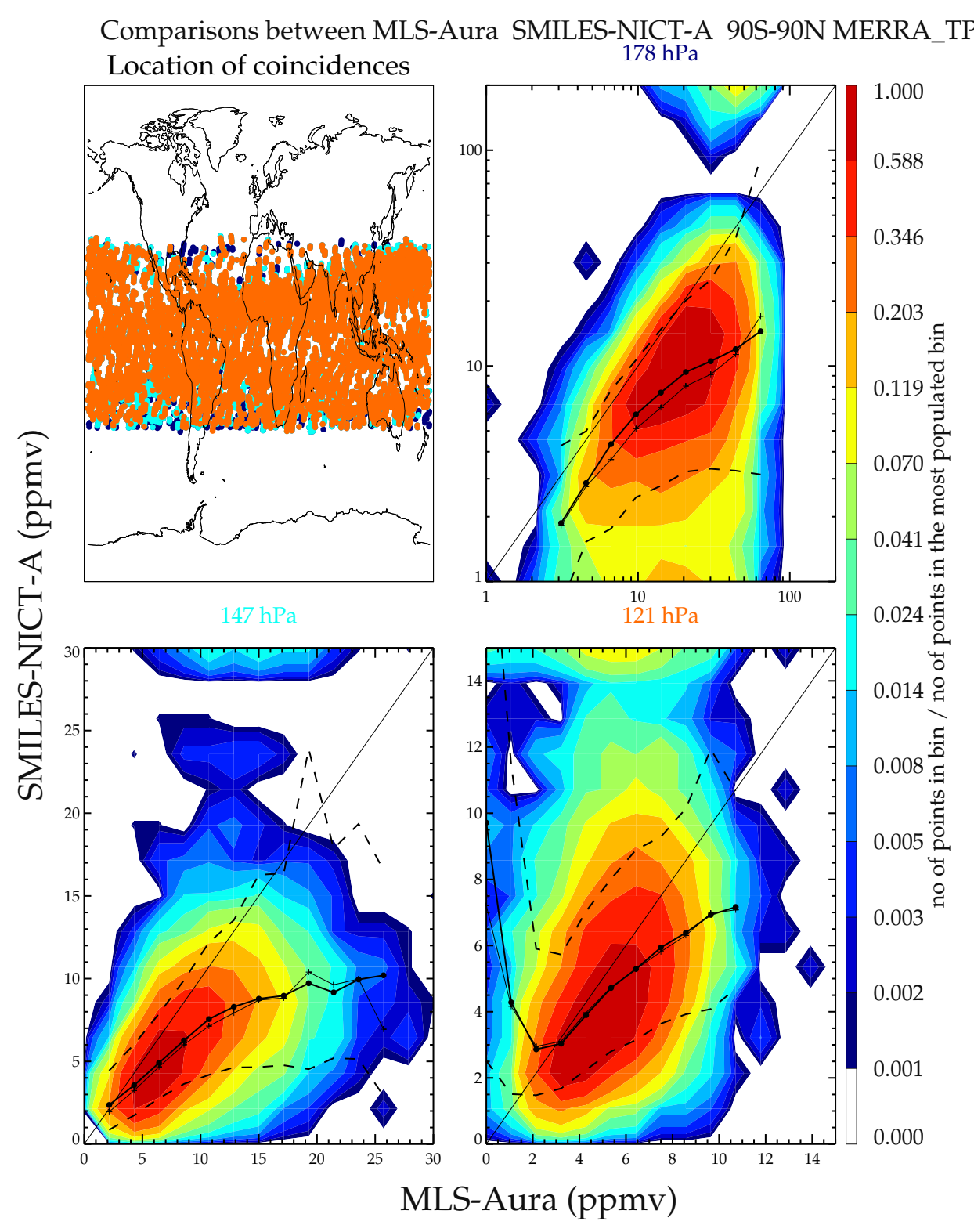

Figure S16. Scatter plot of coincident humidity measurements between MLS-Aura and SMILES NICT A 


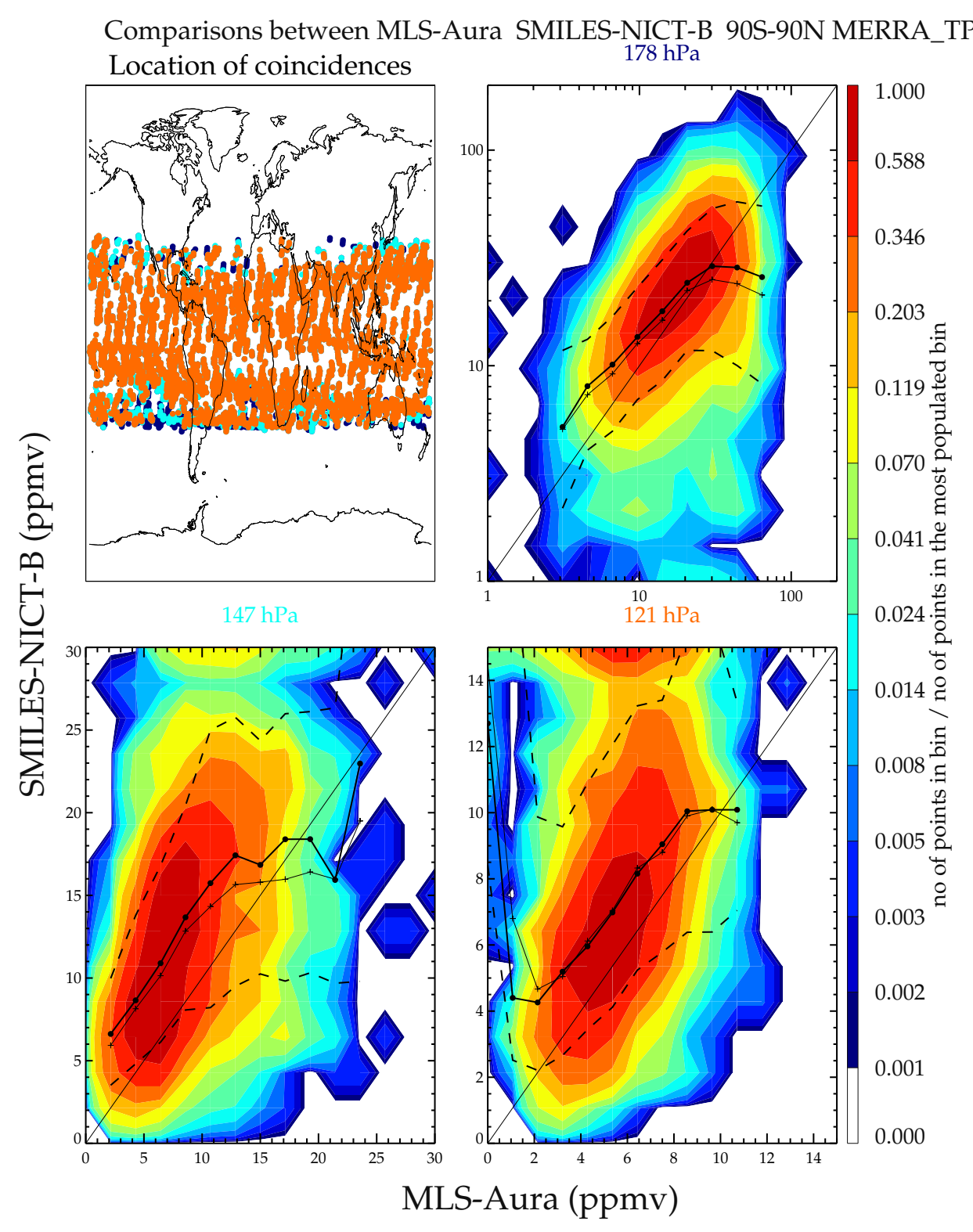

Figure S17. Scatter plot of coincident humidity measurements between MLS-Aura and SMILES NICT B 


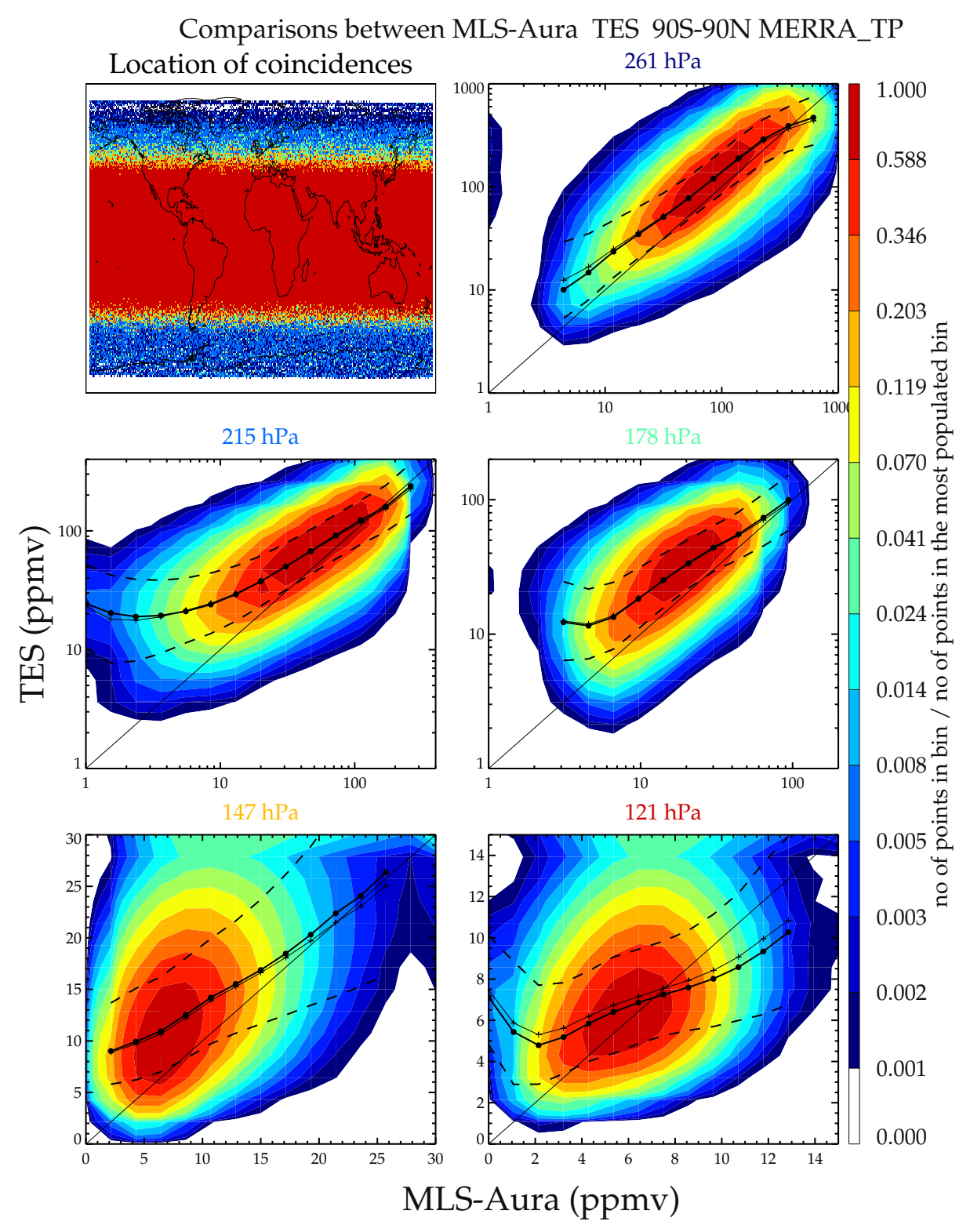

Figure S18. Scatter plot of coincident humidity measurements between MLS-Aura and TES 
Comparisons between MIPAS-ESA ACE-FTS 90S-90N MERRA_TP Location of coincidences

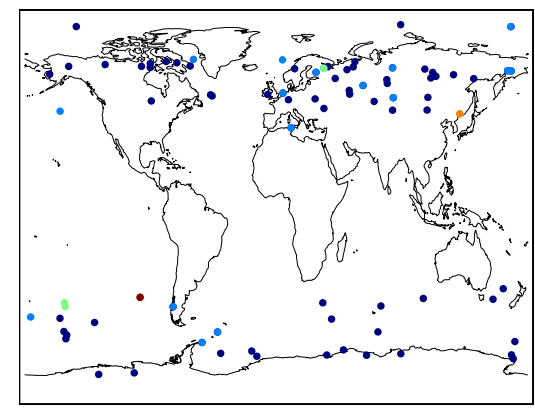
$261.02 \mathrm{hPa}$
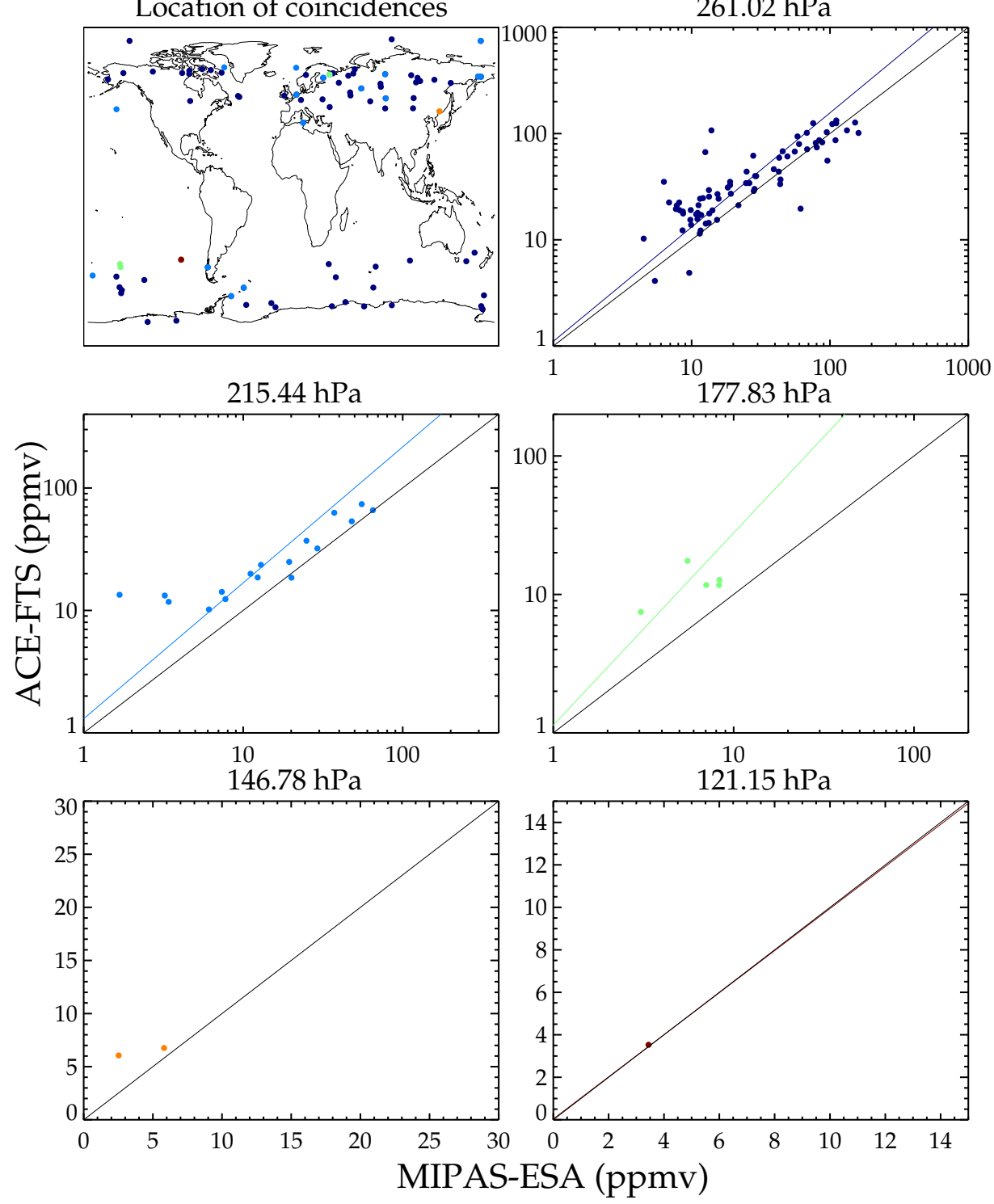

Figure S19. Scatter plot of coincident humidity measurements between MIPAS ESA and ACE-FTS 


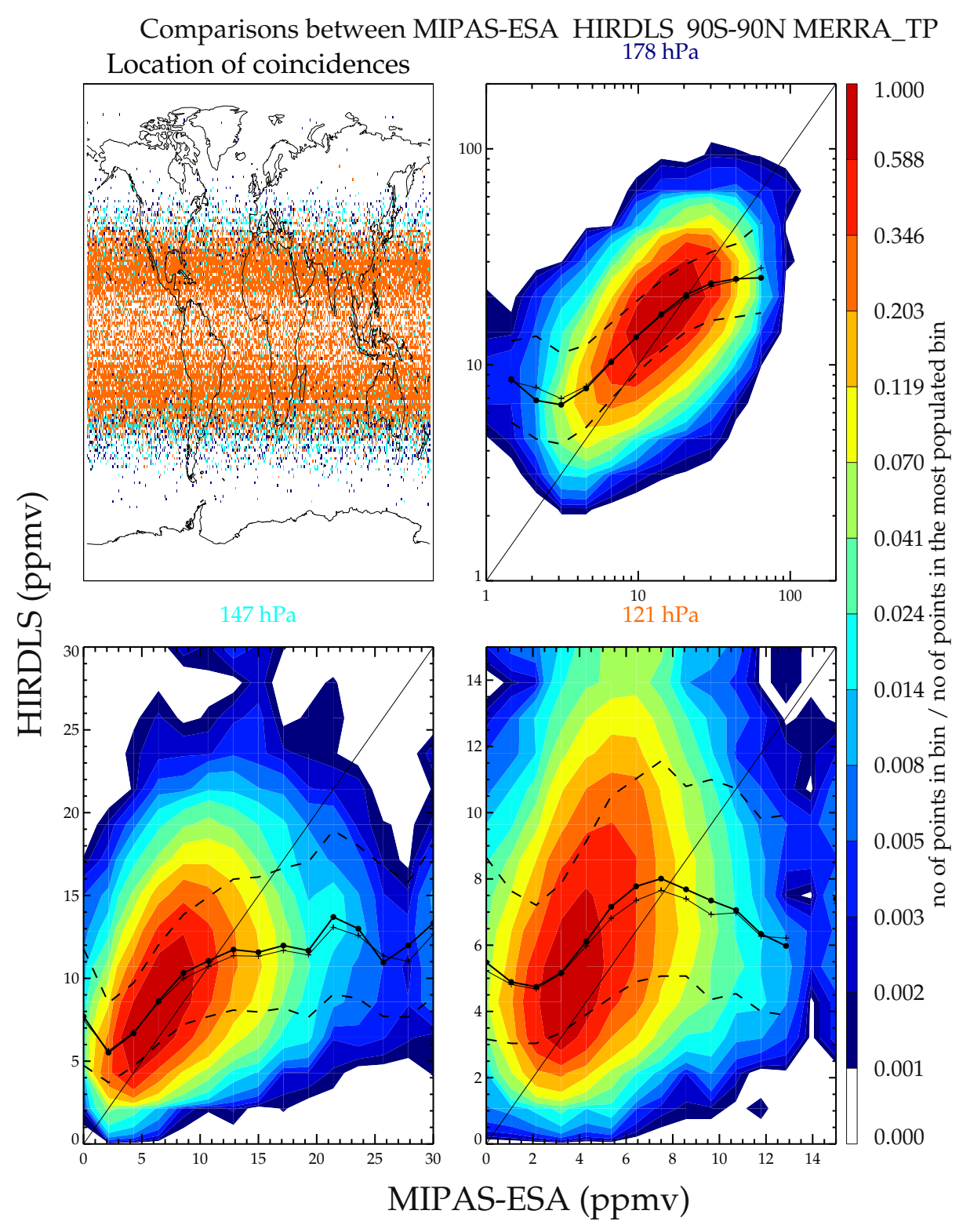

Figure S20. Scatter plot of coincident humidity measurements between MIPAS ESA and HIRDLS 
Comparisons between MIPAS-ESA MIPAS-Bologna 90S-90N MERRA_TP Location of coincidences
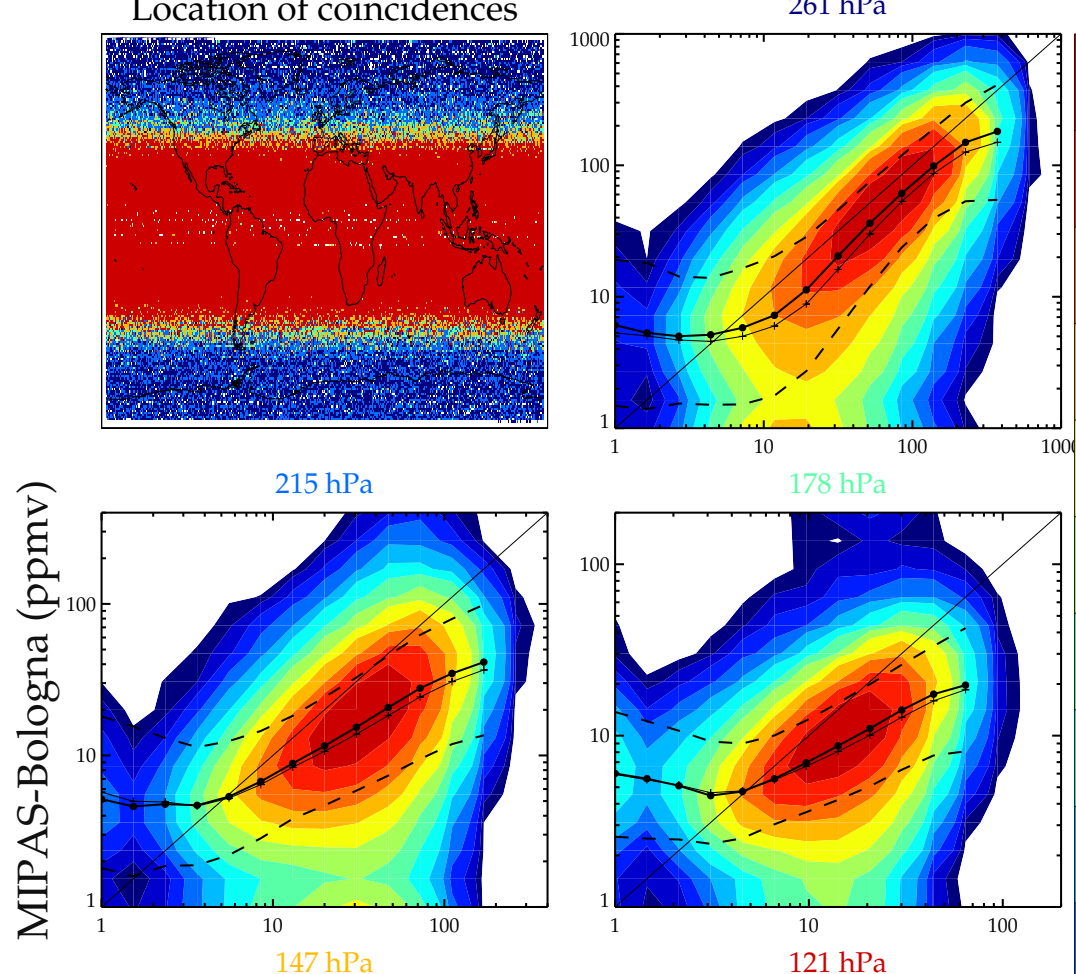

1.000

0.588

0.346

0.203

0.119

0.070 0.0

$0.014 \stackrel{ }{\varrho}$

0.008

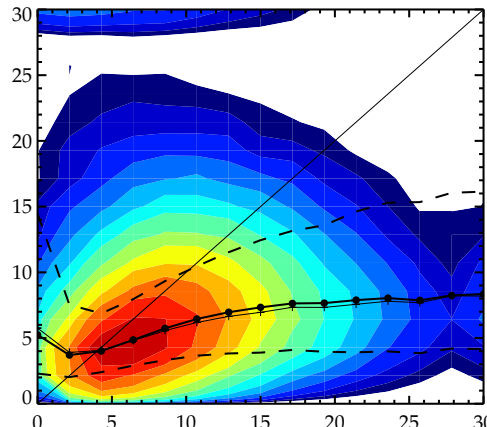

$121 \mathrm{hPa}$

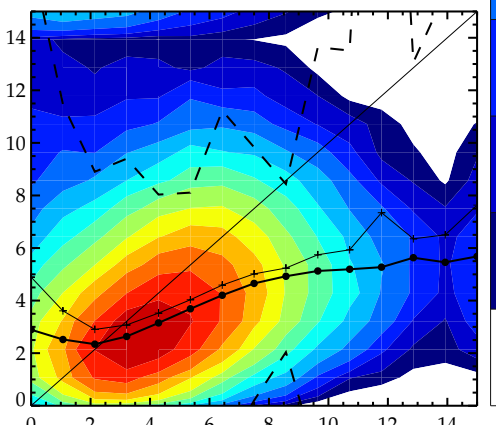

0.005

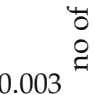

0.002

0.001

MIPAS-ESA (ppmv)

Figure S21. Scatter plot of coincident humidity measurements between MIPAS ESA and MIPAS Bologna 
Comparisons between MIPAS-ESA MIPAS-Oxford 90S-90N MERRA_TP Location of coincidences
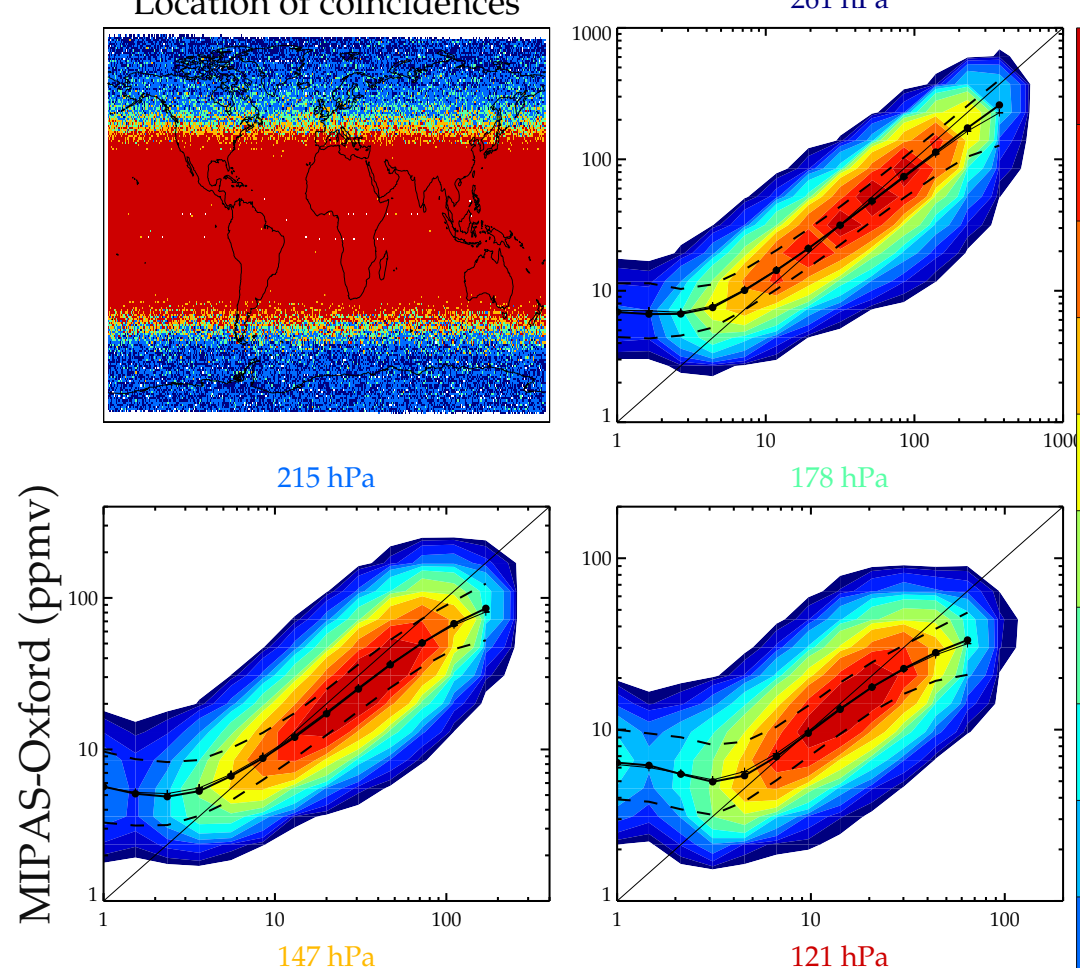

1.000

0.588

0.346

0.203

0.119

(ำ
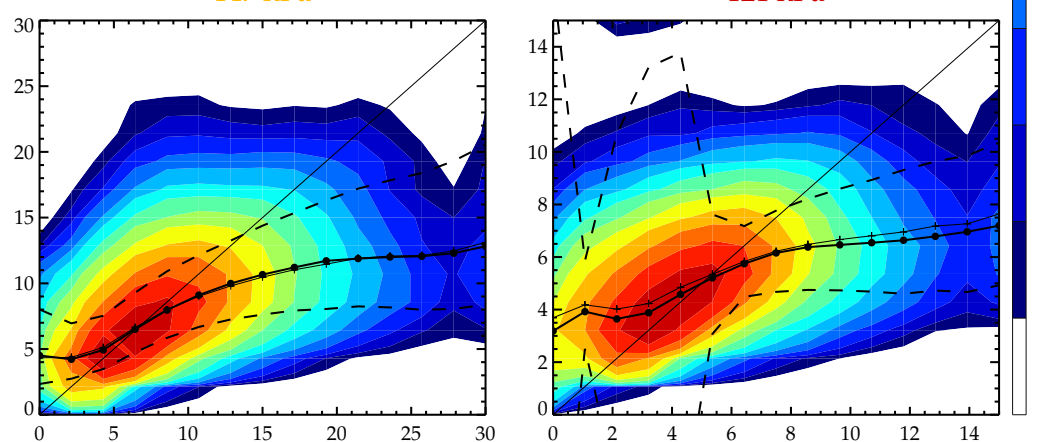
$0.070 \stackrel{\frac{0}{0}}{\frac{0}{0}}$

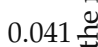

0.024 .

$0.014 \stackrel{\varrho}{\varrho}$

0.008

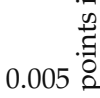

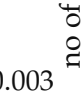

0.002

0.001

MIPAS-ESA (ppmv)

Figure S22. Scatter plot of coincident humidity measurements between MIPAS ESA and MIPAS Oxford 
Comparisons between MIPAS-ESA MIPAS-Oxford 90S-90N MERRA_TP Location of coincidences
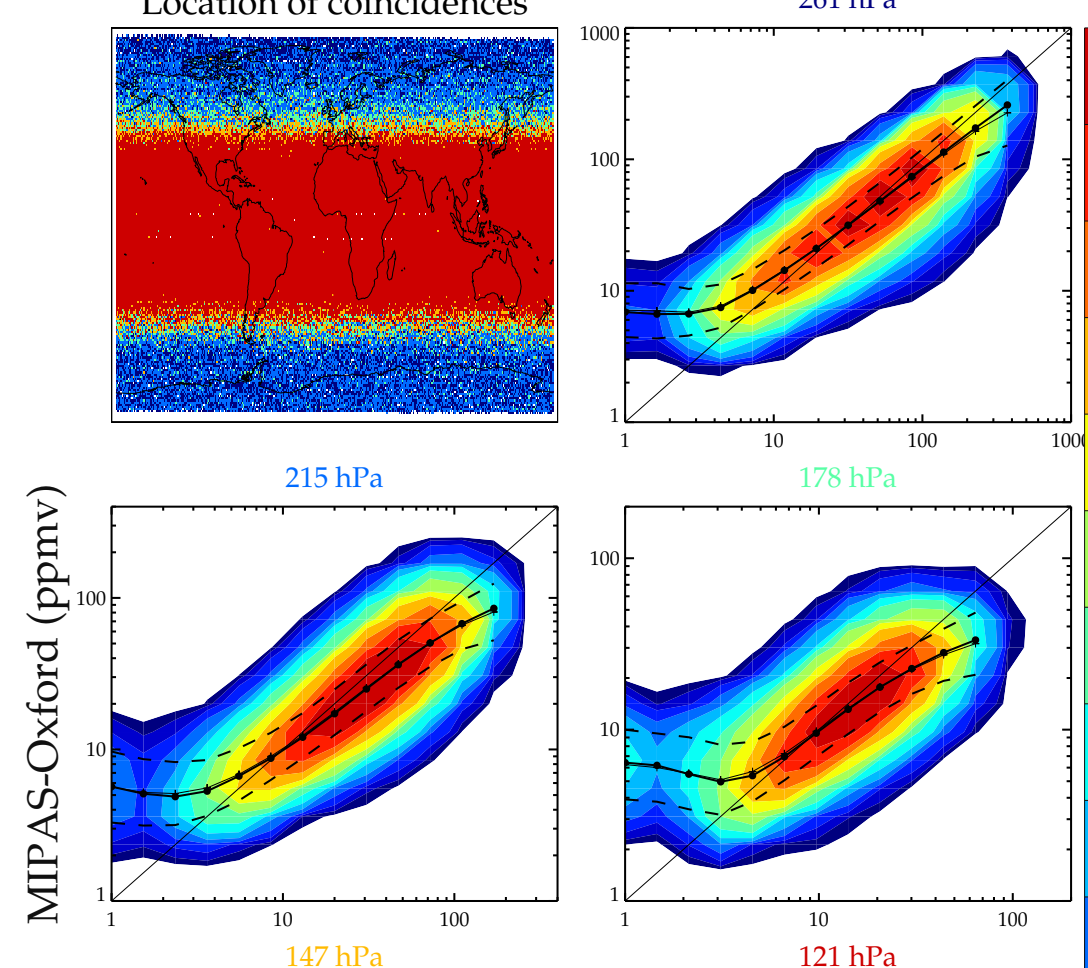

1.000

0.588

0.346

0.203

0.119

(ำ
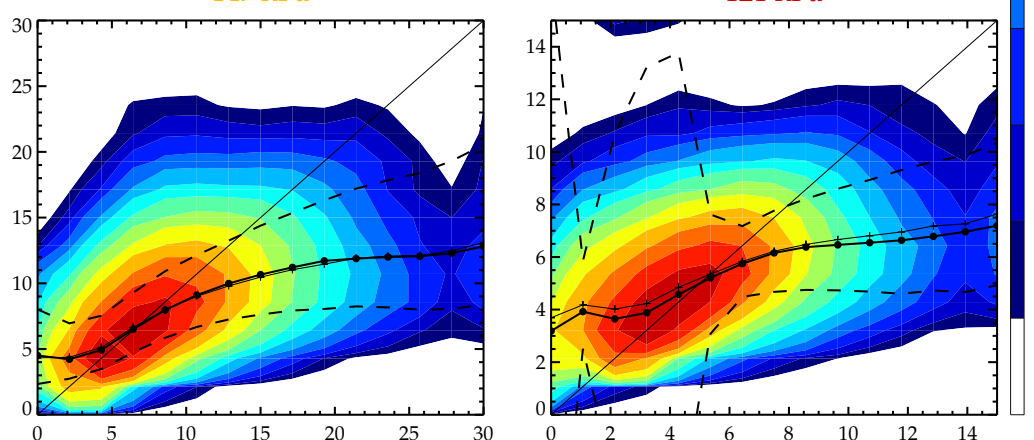
$0.070 \stackrel{\frac{0}{0}}{\frac{0}{0}}$

$0.041 \stackrel{乛}{9}$

0.024 .

$0.014 \stackrel{\varrho}{\varrho}$

0.008

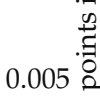

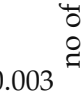

0.002

0.001

MIPAS-ESA (ppmv)

Figure S23. Scatter plot of coincident humidity measurements between MIPAS ESA and MIPAS Oxford 


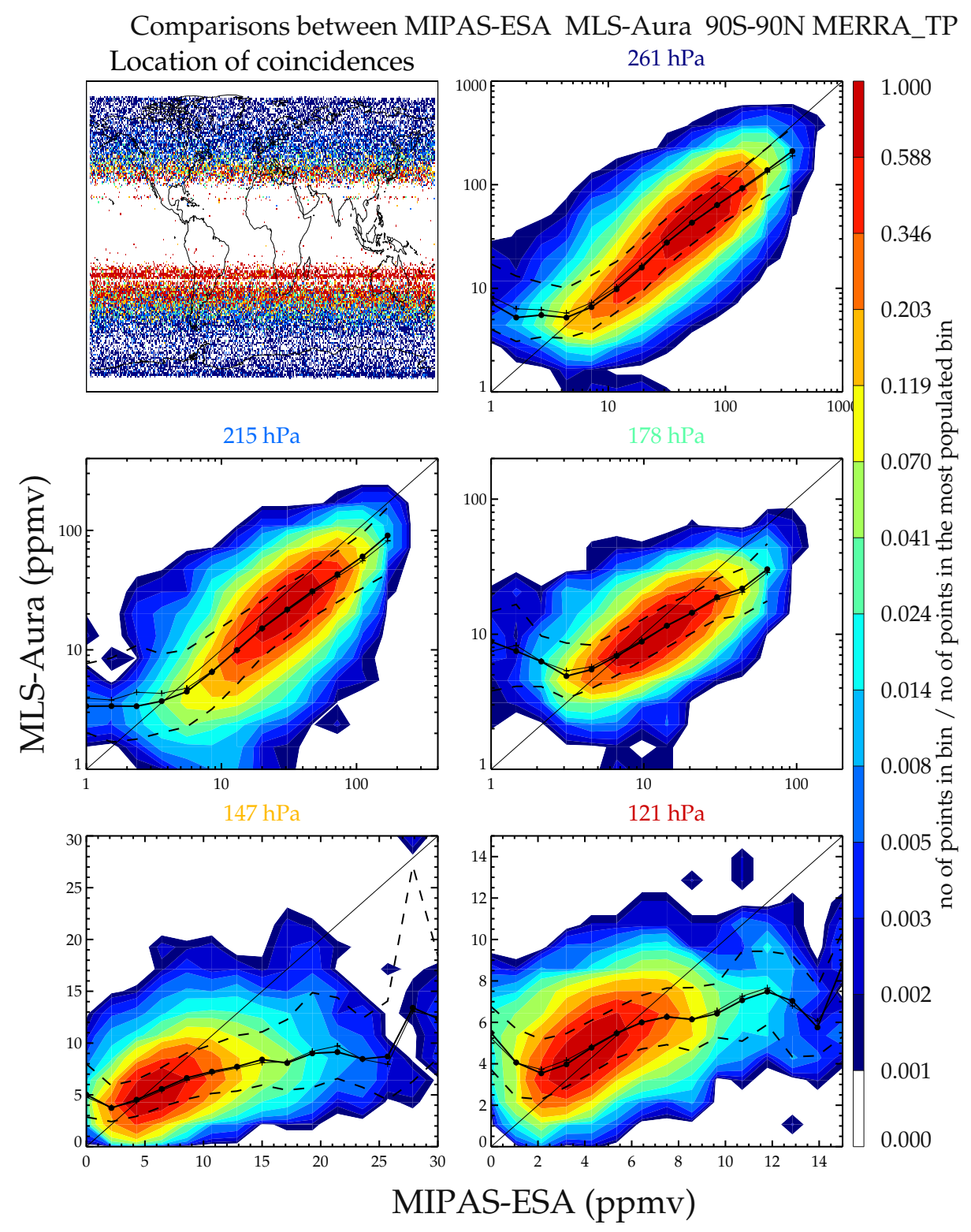

Figure S24. Scatter plot of coincident humidity measurements between MIPAS ESA and MLS Aura 


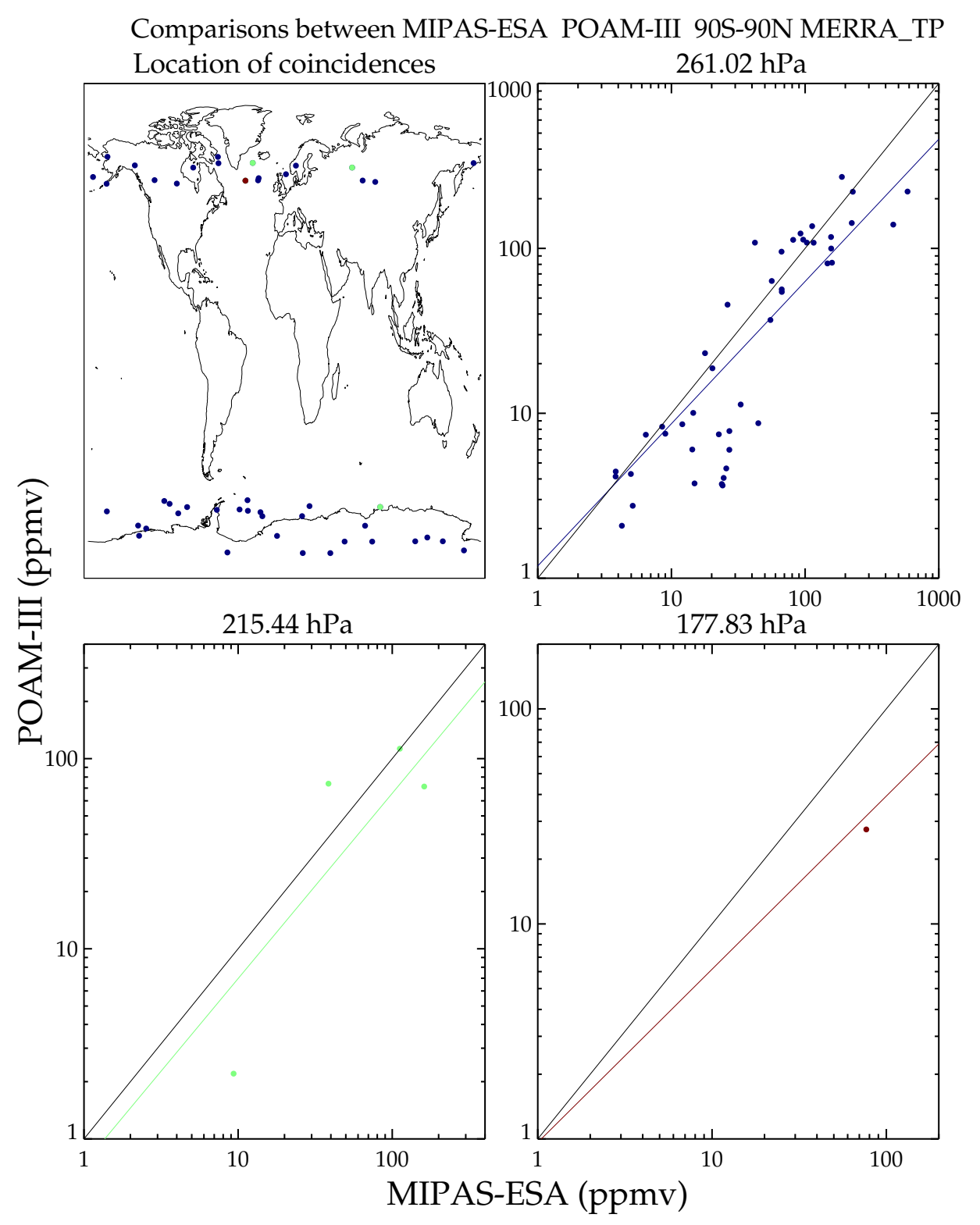

Figure S25. Scatter plot of coincident humidity measurements between MIPAS ESA and POAM-III 


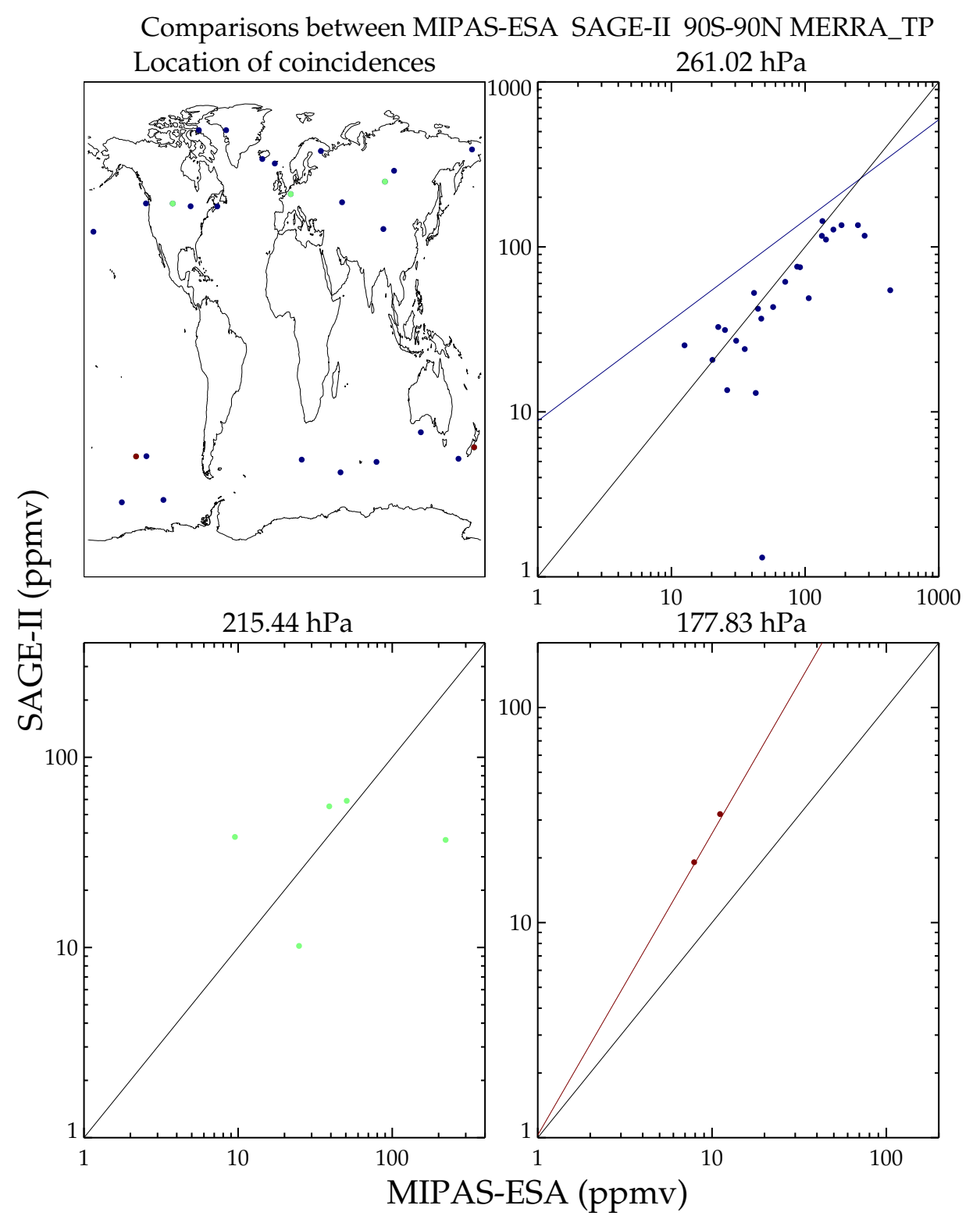

Figure S26. Scatter plot of coincident humidity measurements between MIPAS ESA and SAGE-II 


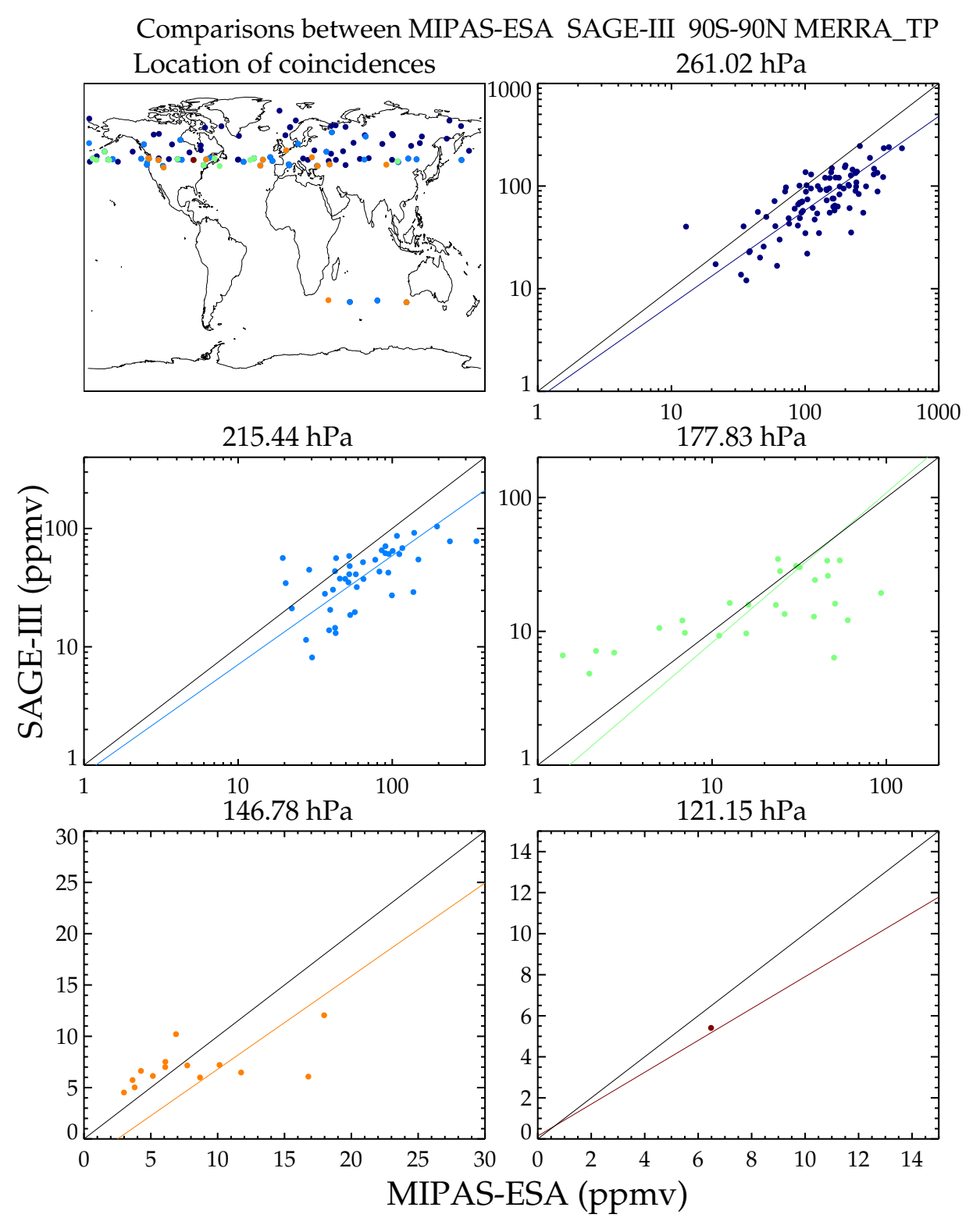

Figure S27. Scatter plot of coincident humidity measurements between MIPAS ESA and SAGE-III 
Comparisons between MIPAS-ESA SCIAMACHY 90S-90N MERRA_TP Location of coincidences
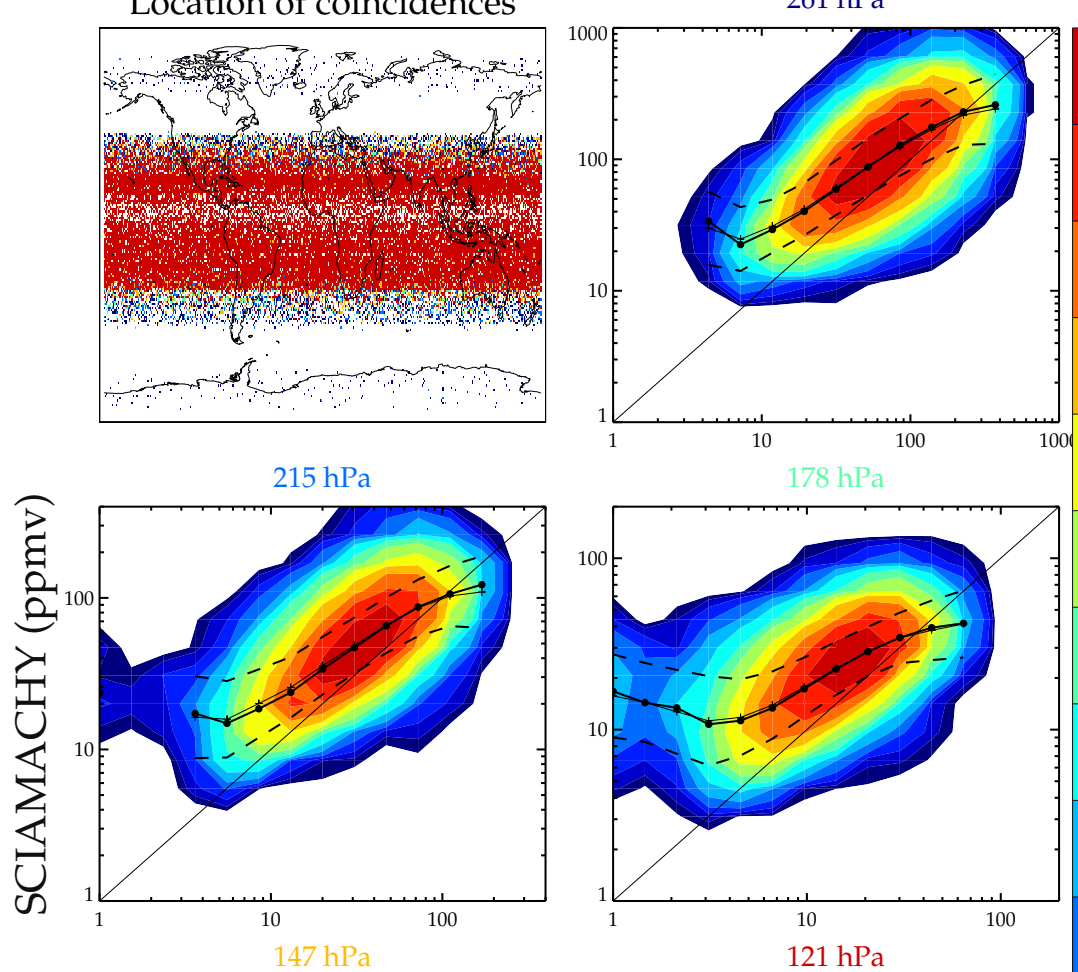

1.000

0.588

0.346

0.203

0.119

$\frac{\pi}{3}$
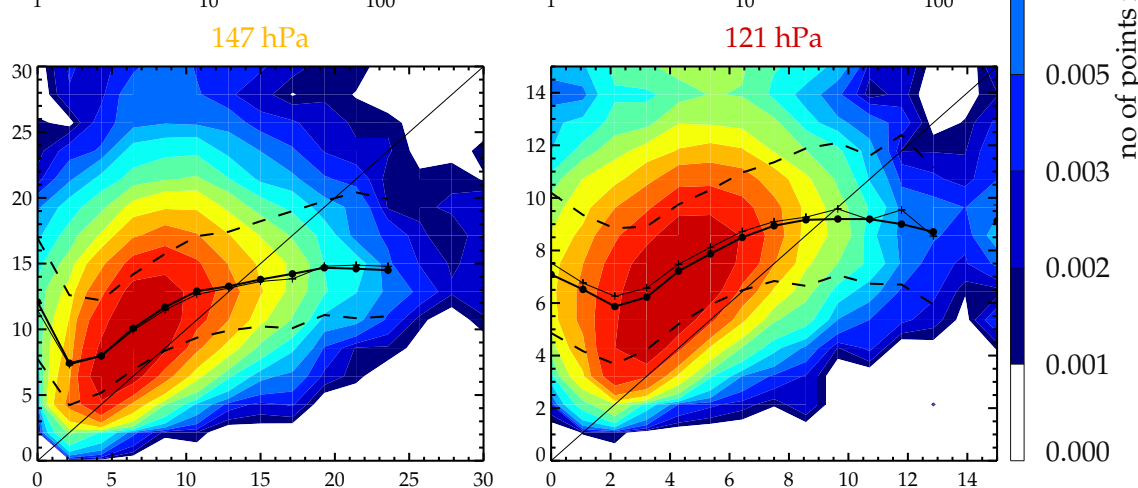

MIPAS-ESA (ppmv)

Figure S28. Scatter plot of coincident humidity measurements between MIPAS ESA and SCIAMACHY 
Comparisons between MIPAS-ESA SMR 90S-90N MERRA_TP Location of coincidences
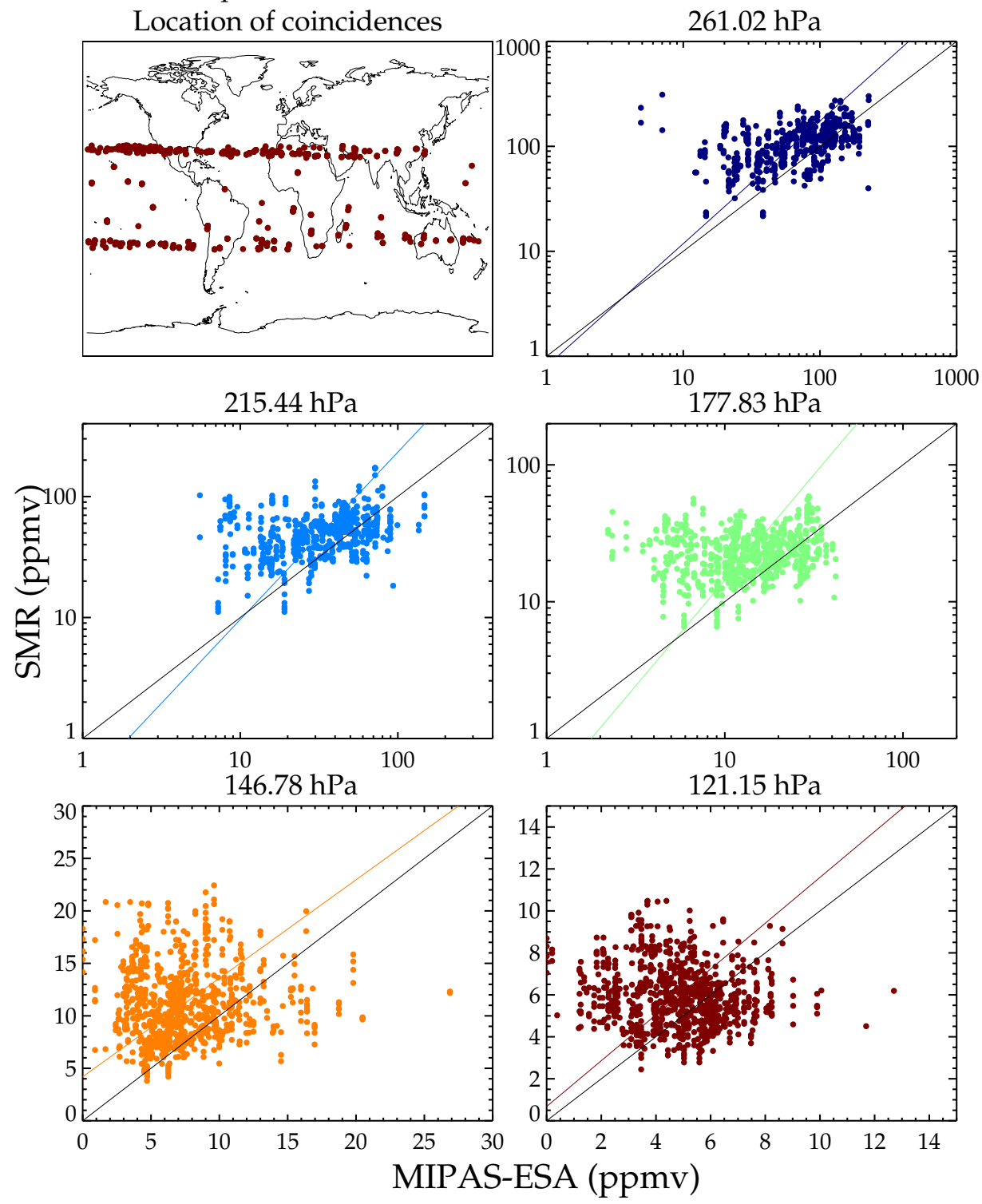

Figure S29. Scatter plot of coincident humidity measurements between MIPAS ESA and SMR 
Comparisons between MIPAS-ESA TES 90S-90N MERRA_TP
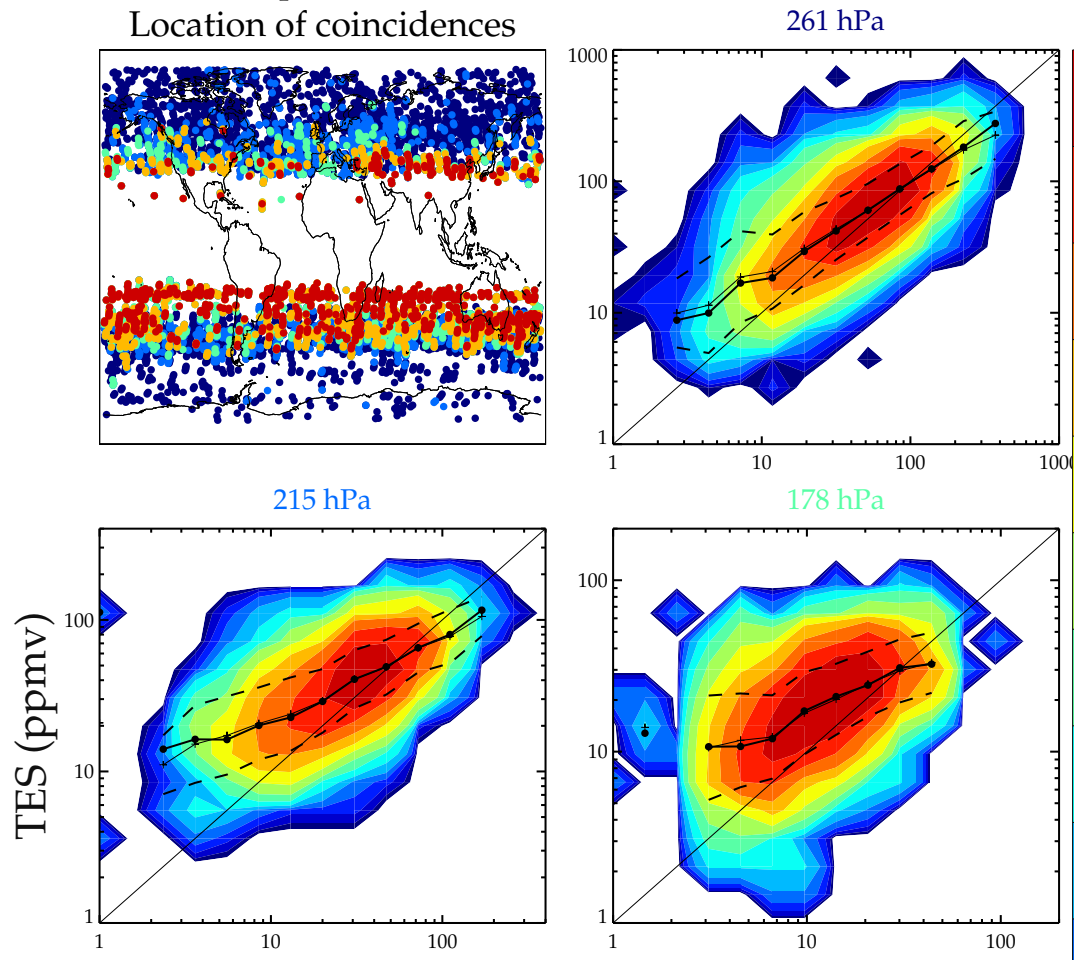

1.000

0.588

0.346

0.203

0.119

范 $0.070 \frac{\stackrel{2}{0}}{\text { की }}$

$0.041 \stackrel{\oplus}{\mathscr{*}}$

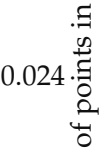

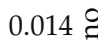

0.008 ق

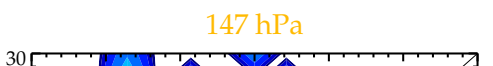

$121 \mathrm{hPa}$
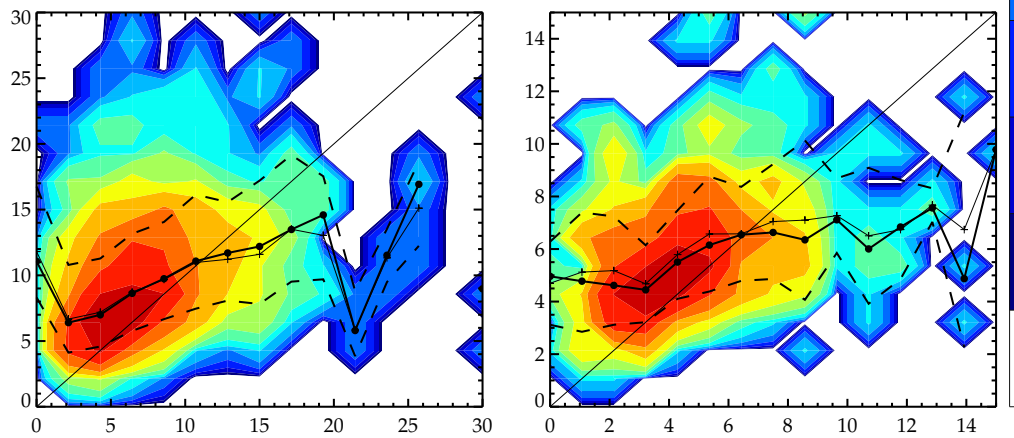

0.005

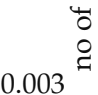

0.002

0.001

MIPAS-ESA (ppmv)

Figure S30. Scatter plot of coincident humidity measurements between MIPAS ESA and TES 


\subsubsection{1-2008.02.29 $\mathrm{p}=250 \mathrm{hPa}$}
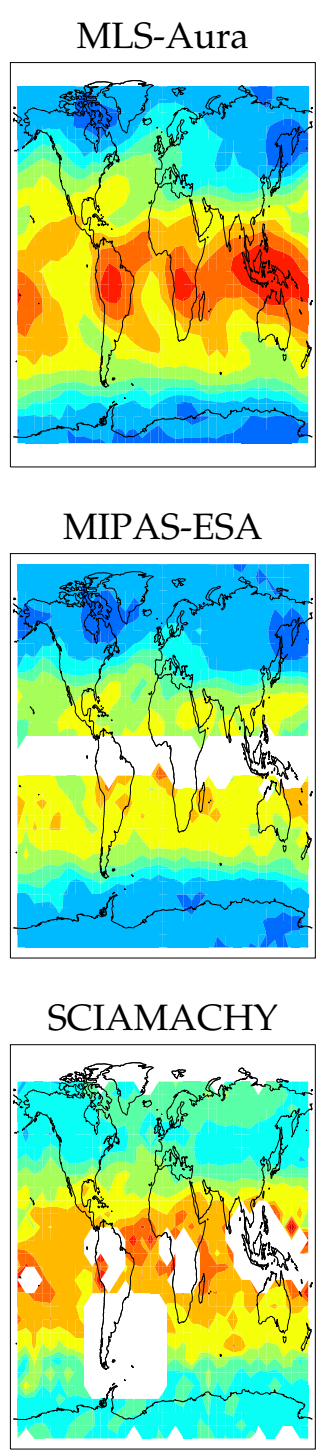
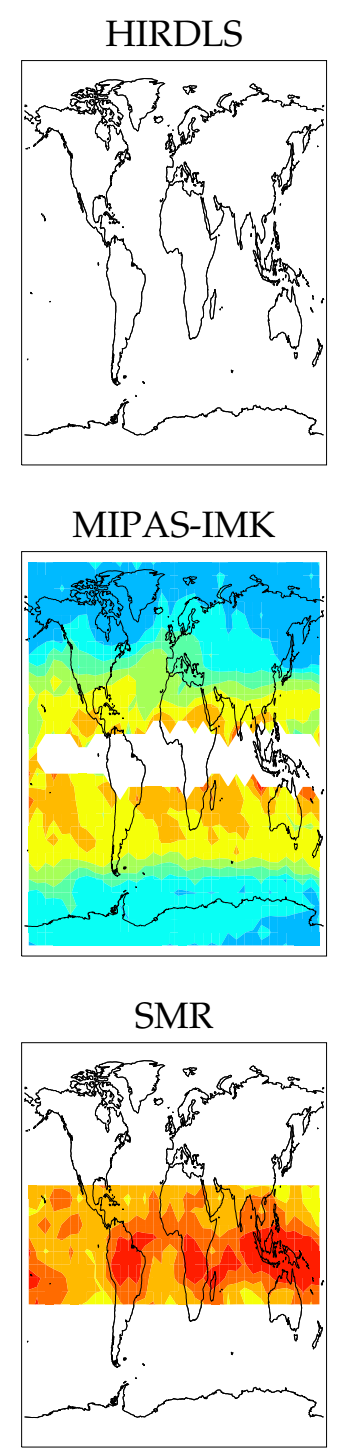
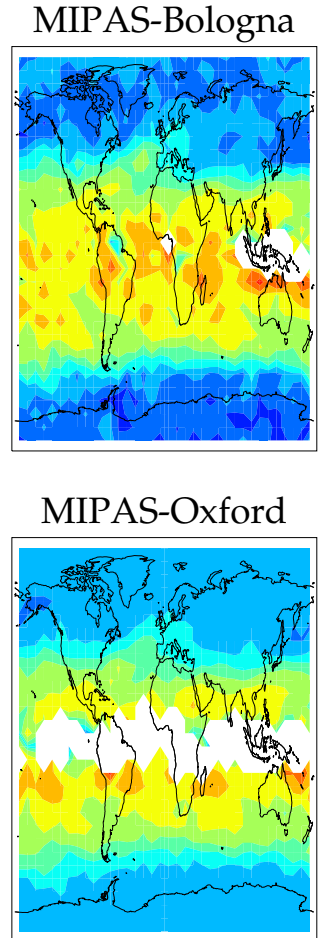

138.95

84.83

51.79

31.62 䒕

19.31

11.79

7.20

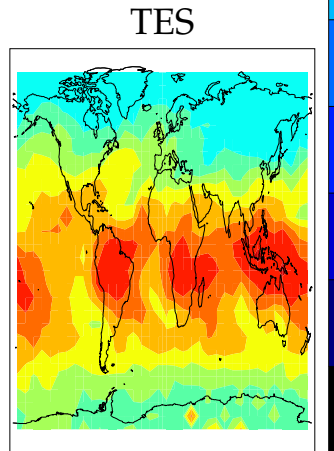

Figure S31. Gridded maps for nine instruments at $250 \mathrm{hPa}$. 


\subsubsection{1-2008.02.29 $\mathrm{p}=200 \mathrm{hPa}$}
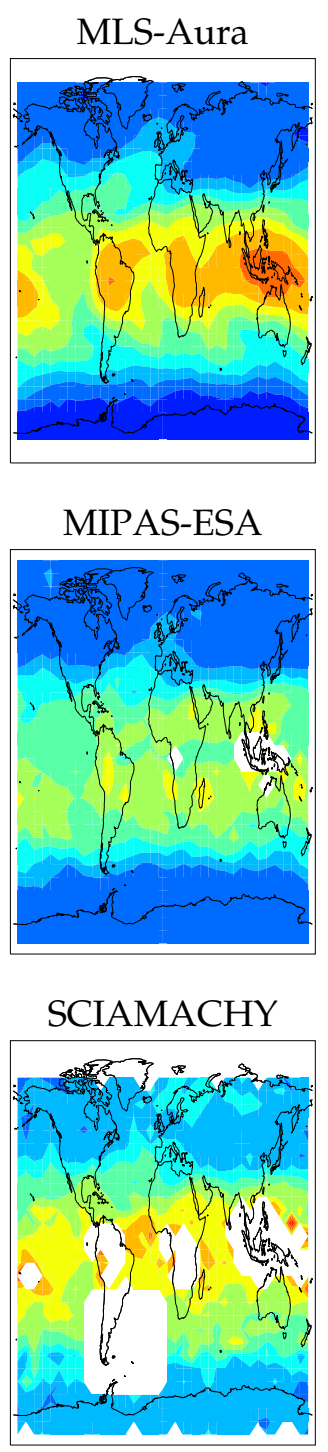
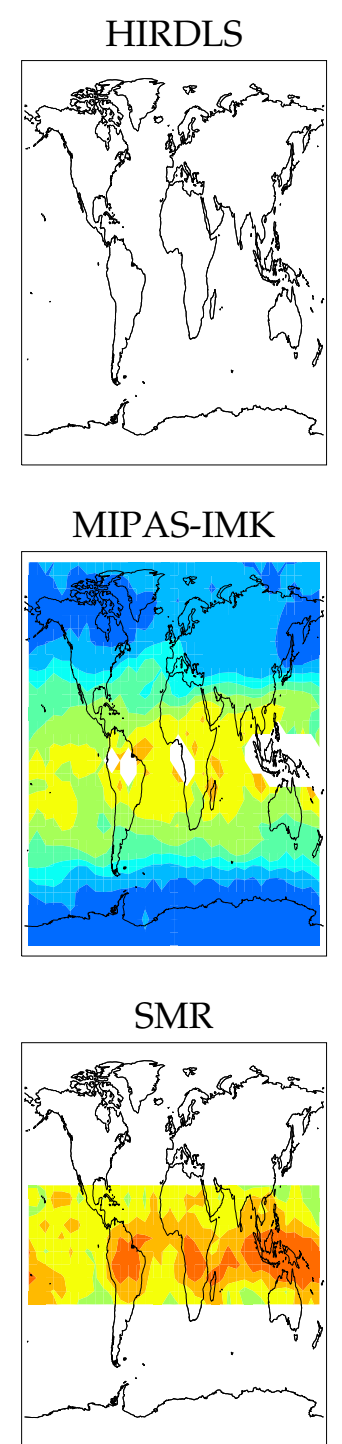

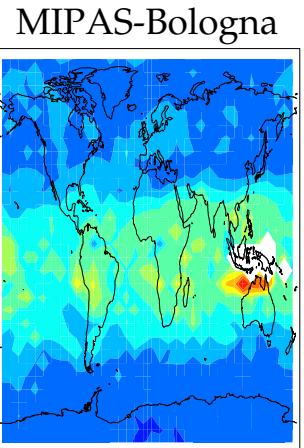

500.00

320.76

205.78

132.01

84.69

54.33

34.86

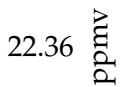

14.35

9.20

5.90

3.79

2.43

1.56

1.00

Figure S32. Gridded maps for nine instruments at $200 \mathrm{hPa}$. 


\subsubsection{1-2008.02.29 $\mathrm{p}=150 \mathrm{hPa}$}
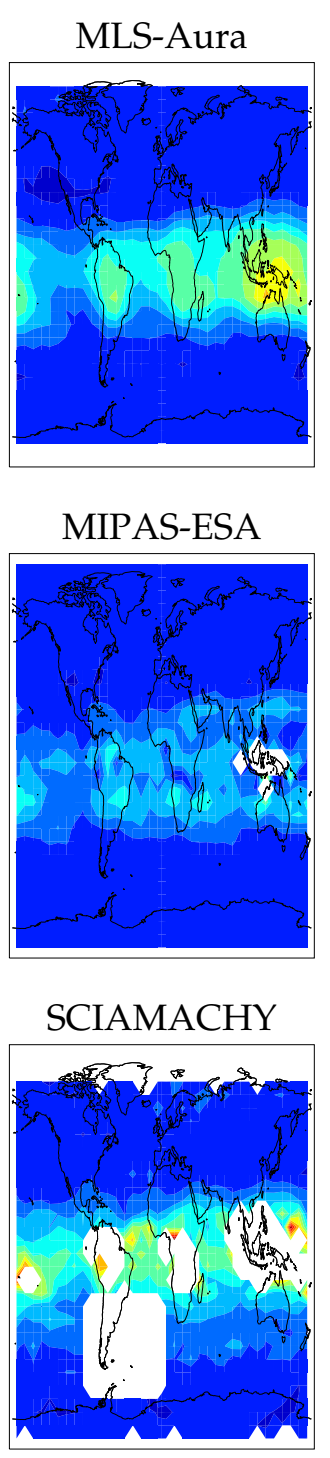
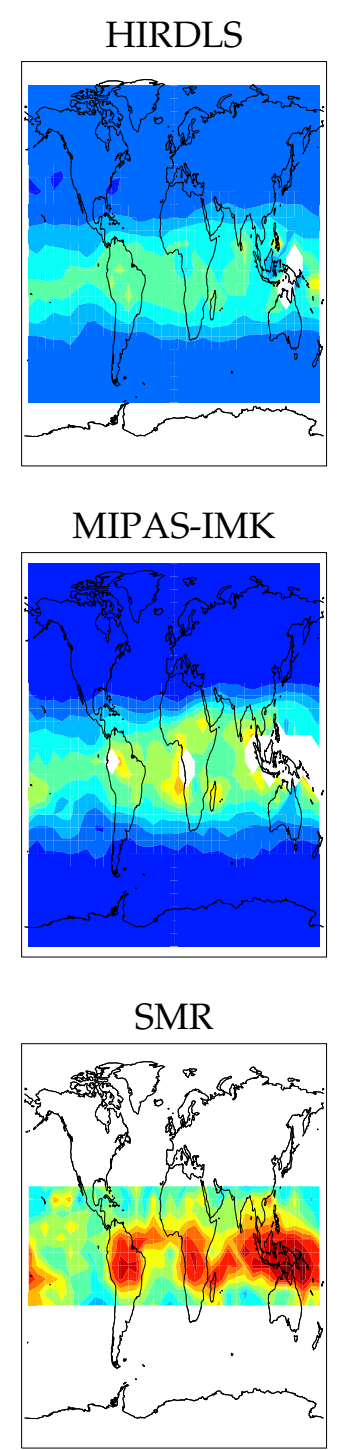

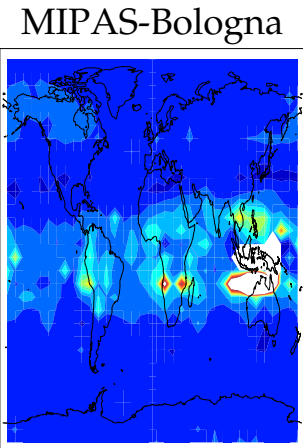

25.00

23.21

21.43

19.64

17.86

MIPAS-Oxford

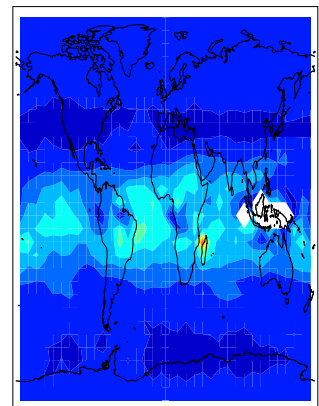

16.07

14.29

12.50 है ڤี

10.71

8.93

7.14

5.36

3.57

1.79

0.00

Figure S33. Gridded maps for nine instruments at $150 \mathrm{hPa}$. 
Mapped field grid scatter 2007.12.01-2008.02.29
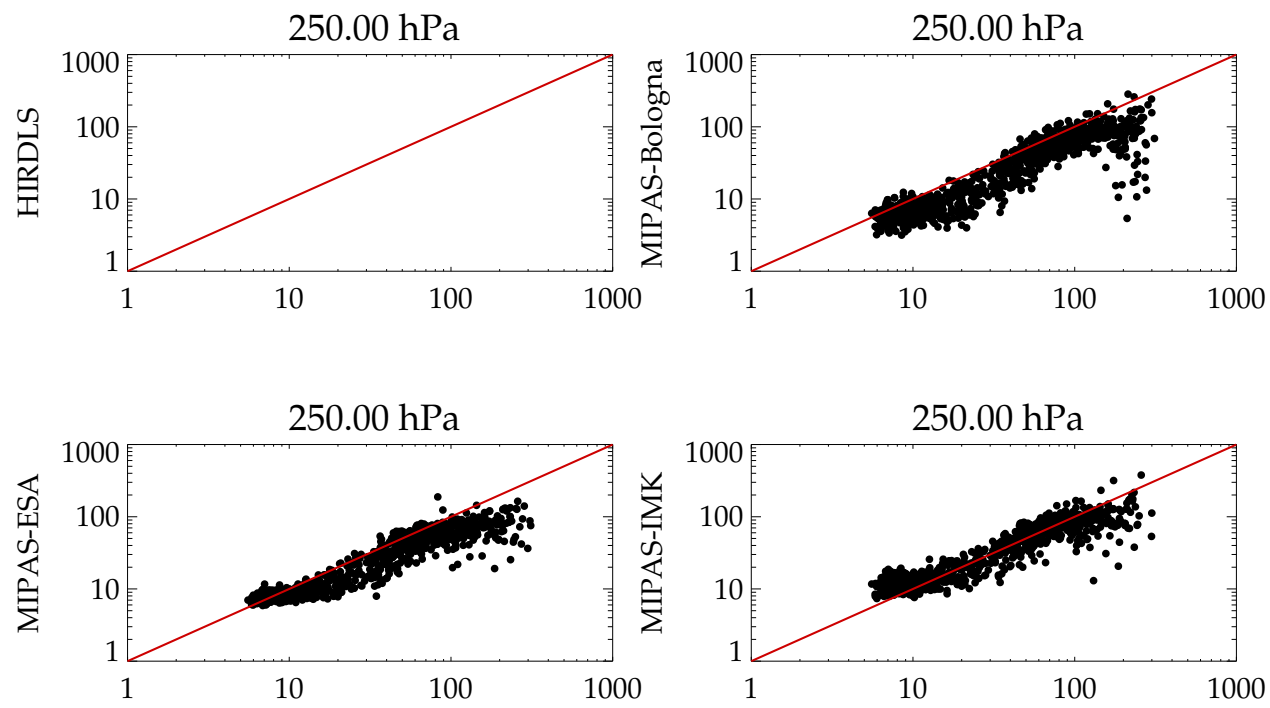

己े
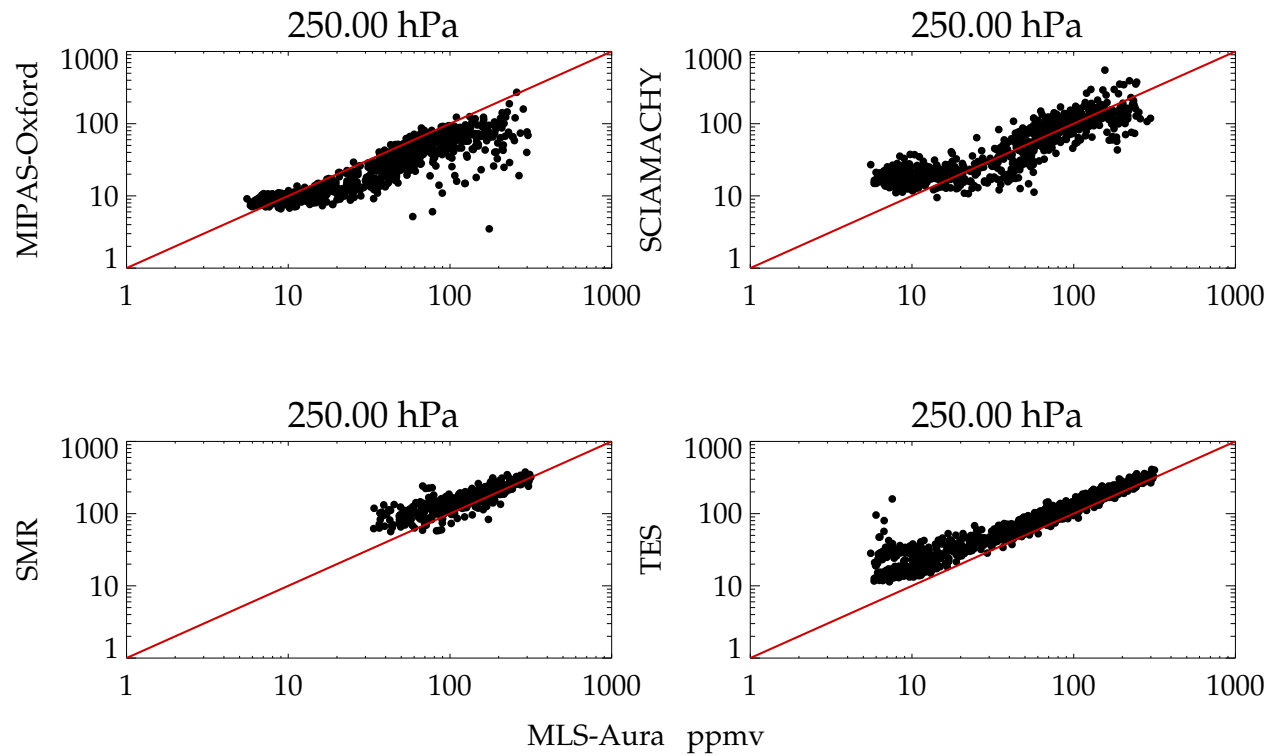

Figure S34. Scatter plot of mapped grid box values of 8 instruments versus MLS Aura at $250 \mathrm{hPa}$. 
Mapped field grid scatter 2007.12.01-2008.02.29
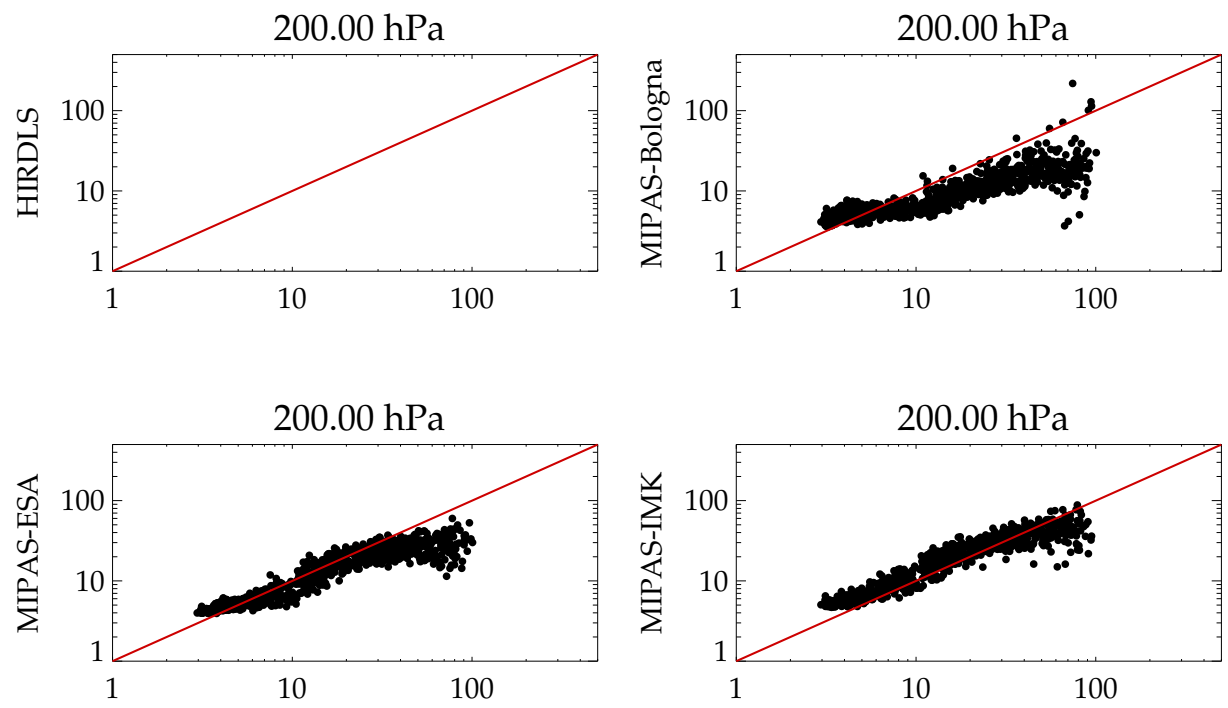

己े
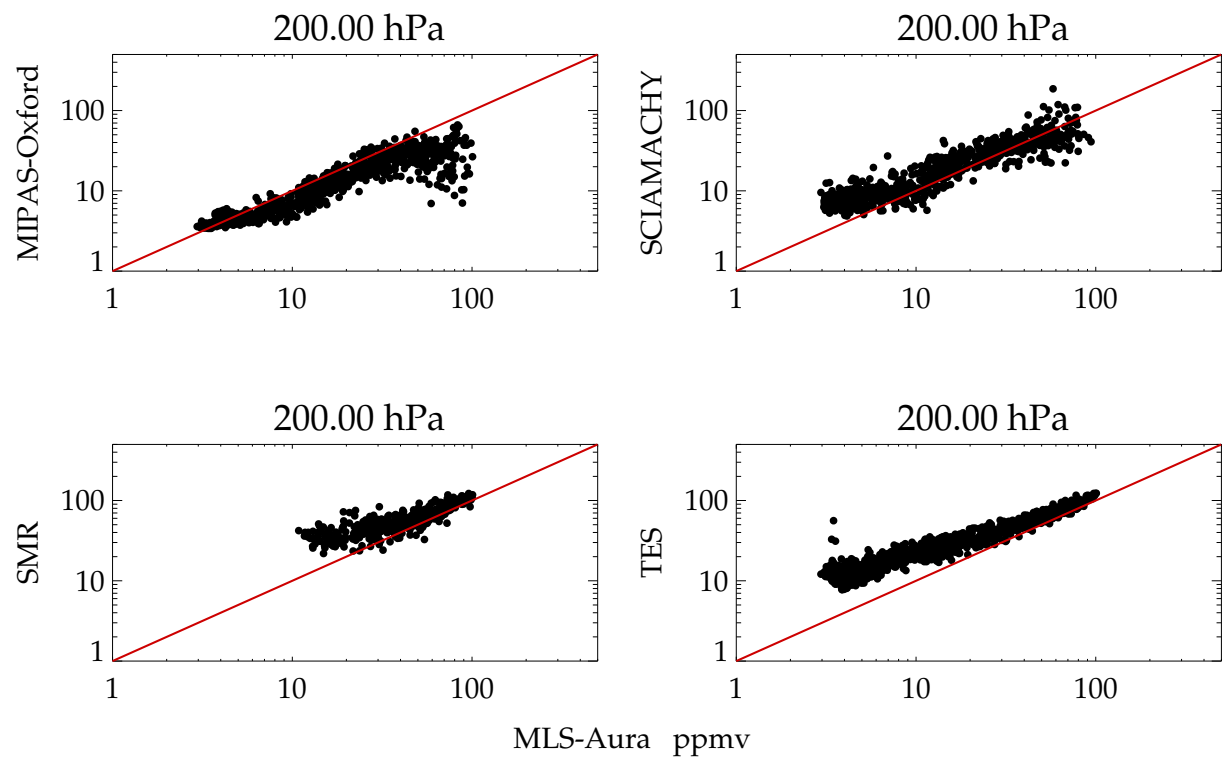

Figure S35. Scatter plot of mapped grid box values of 8 instruments versus MLS Aura at $200 \mathrm{hPa}$. 
Mapped field grid scatter 2007.12.01-2008.02.29
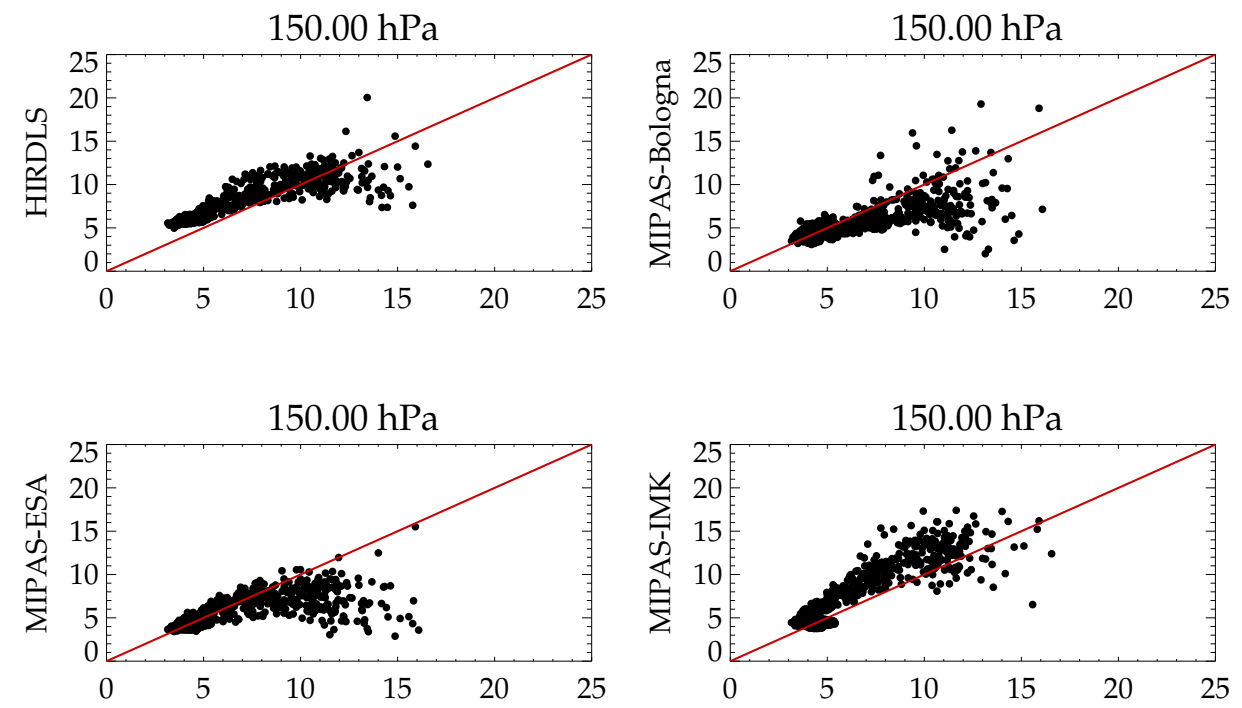

己े
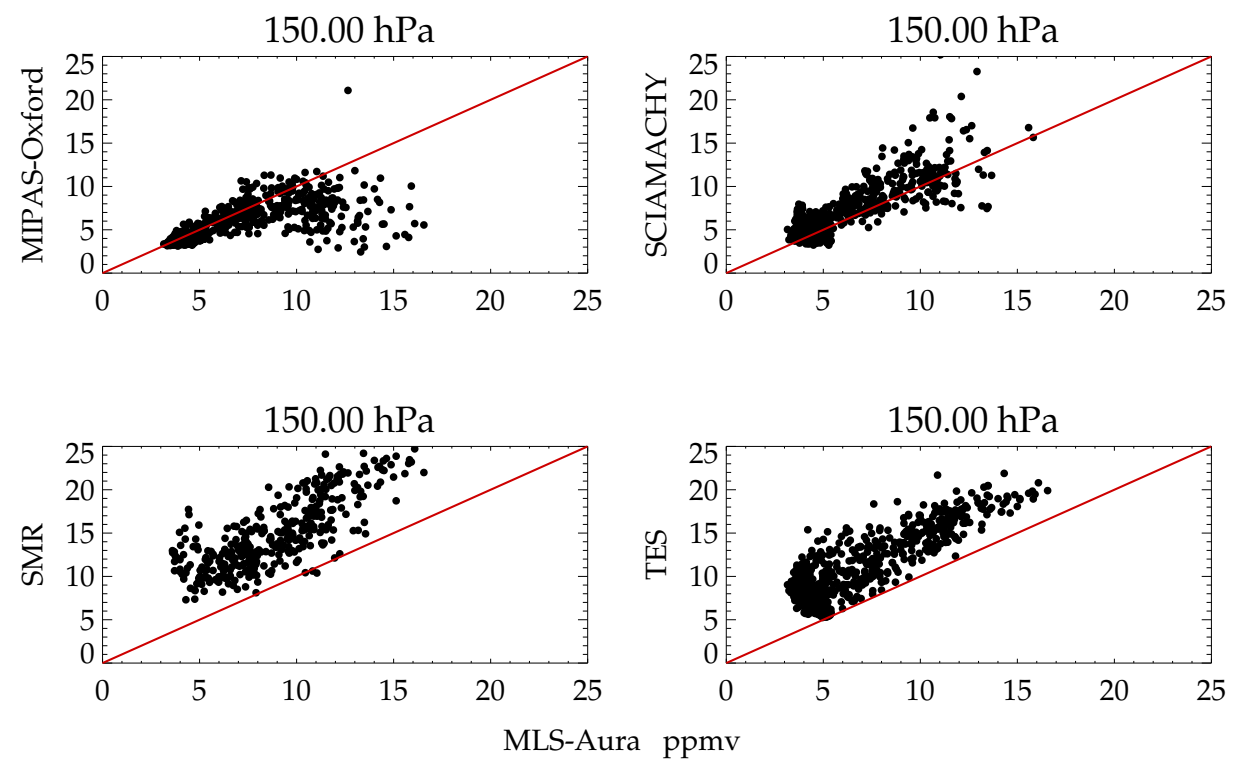

Figure S36. Scatter plot of mapped grid box values of 8 instruments versus MLS Aura at $150 \mathrm{hPa}$. 


\subsubsection{1-2010.03.31 $\mathrm{p}=250 \mathrm{hPa}$}
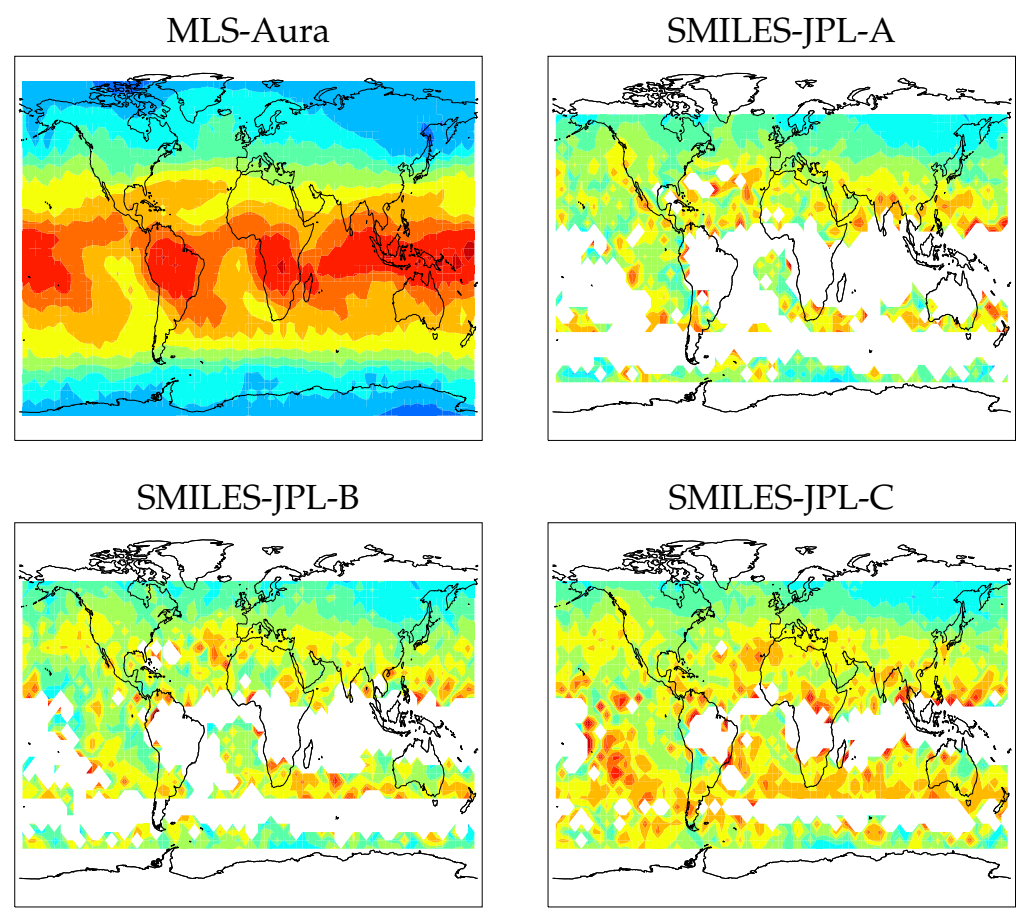

138.95

84.83

51.79

31.62 है

19.31

11.79
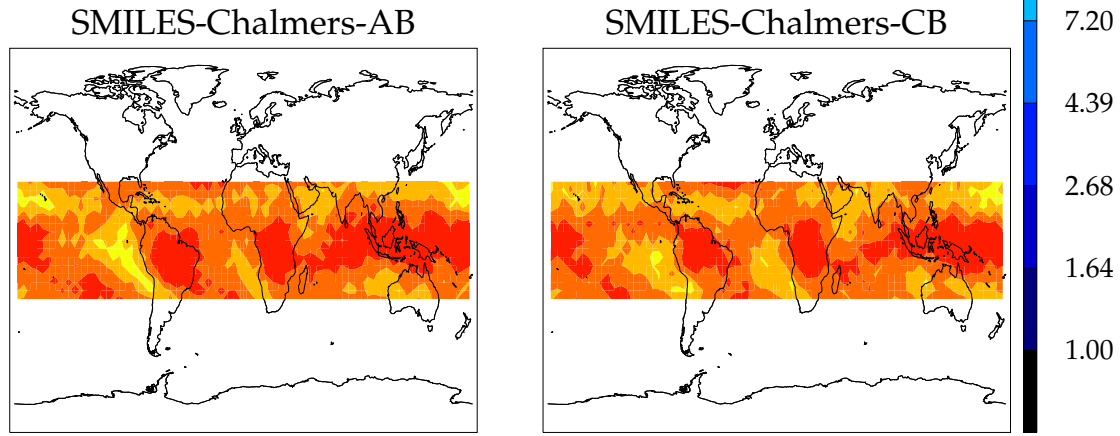

Figure S37. Gridded maps for SMILES humidity retrieval products at $250 \mathrm{hPa}$. 


\subsubsection{1-2010.03.31 $\mathrm{p}=200 \mathrm{hPa}$}
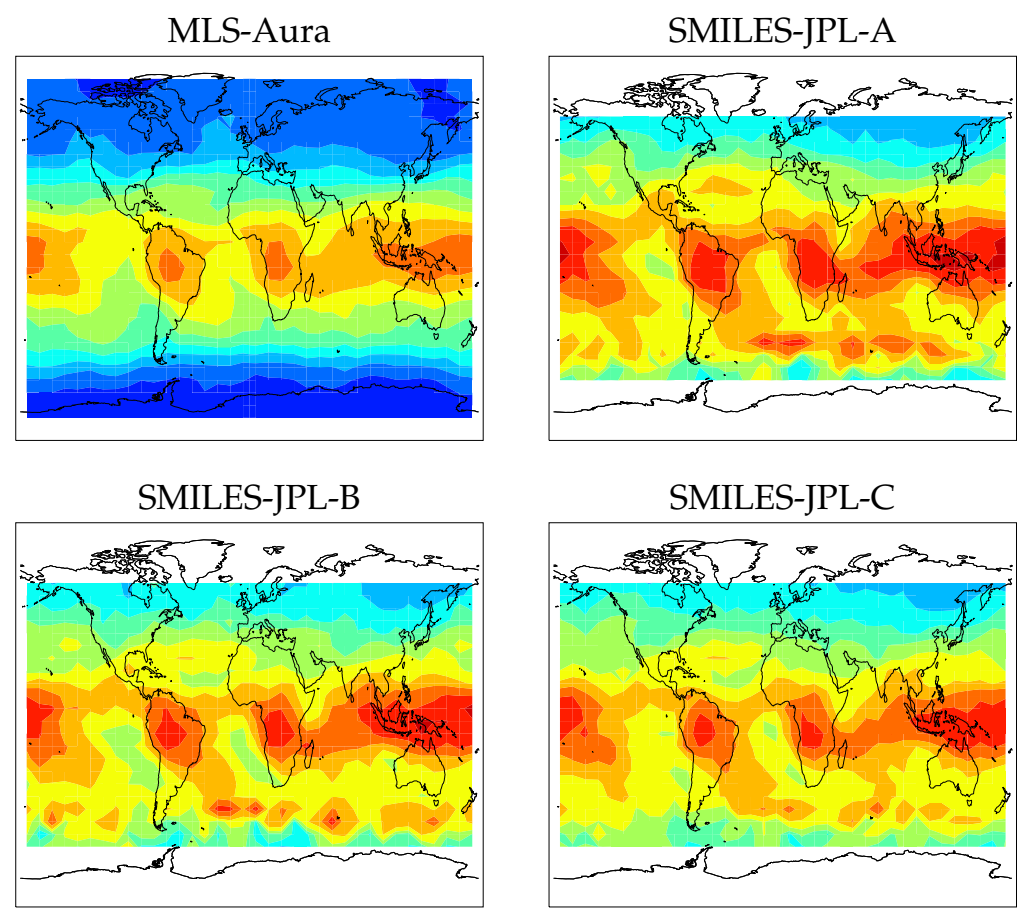

84.69

54.33

34.86

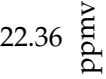

14.35

9.20
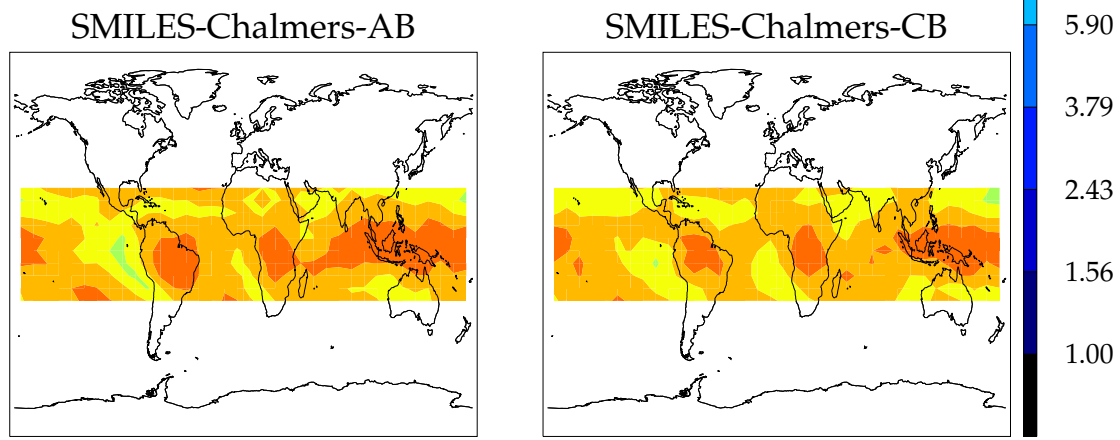

Figure S38. Gridded maps for SMILES humidity retrieval products at $200 \mathrm{hPa}$. 


\subsubsection{1-2010.03.31 $\mathrm{p}=150 \mathrm{hPa}$}
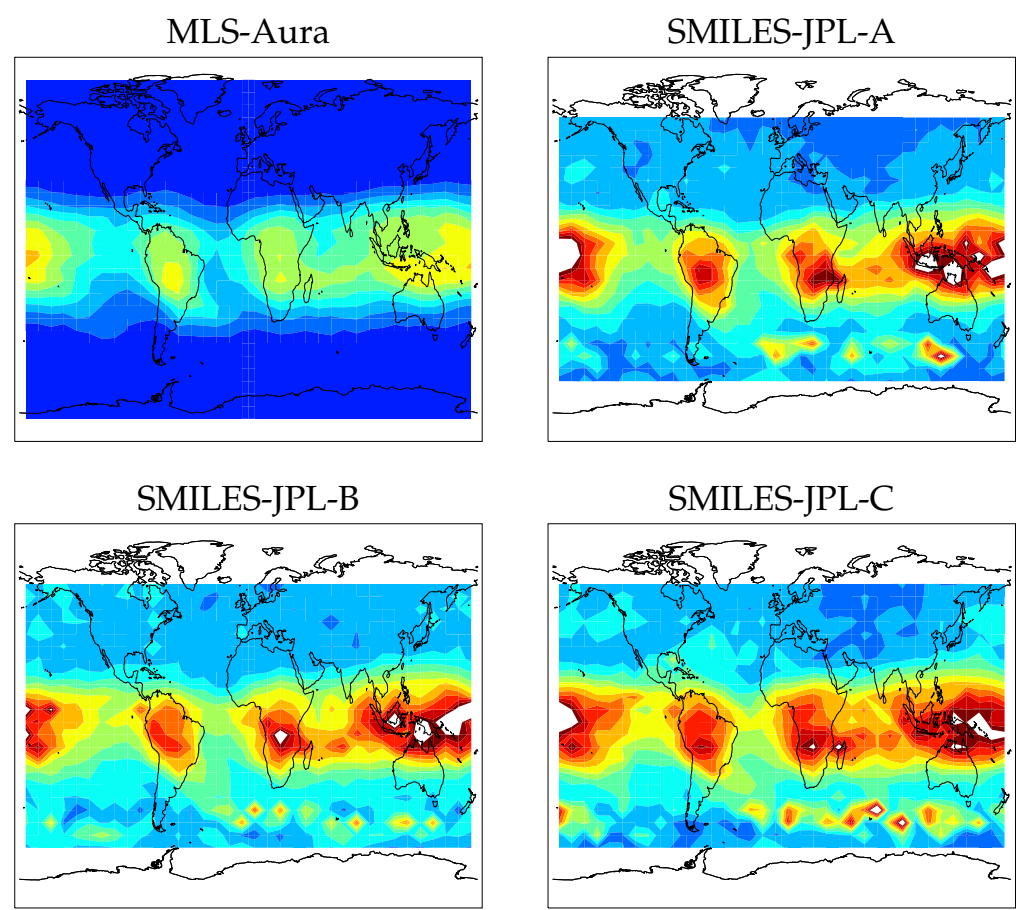

17.86

16.07

14.29

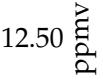

10.71

8.93
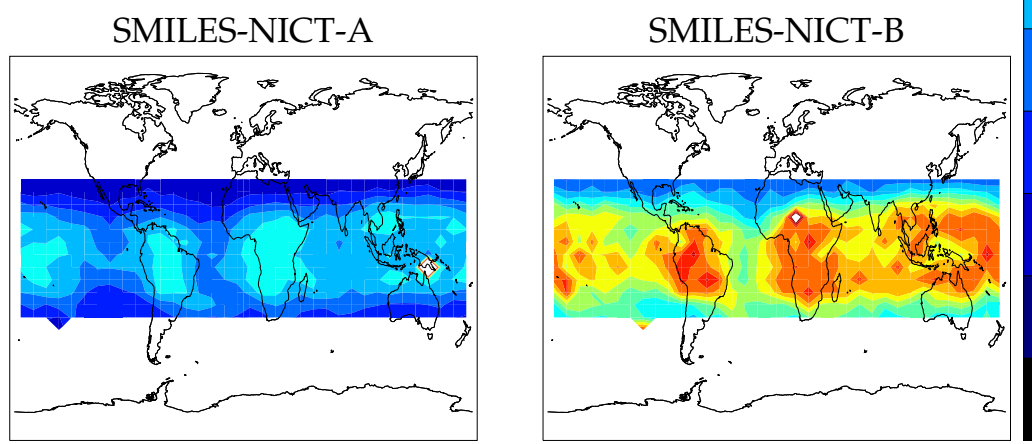

7.14

5.36

3.57

1.79

0.00

Figure S39. Gridded maps for SMILES humidity retrieval products at $150 \mathrm{hPa}$. 
Mapped field grid scatter 2009.12.01-2010.03.31
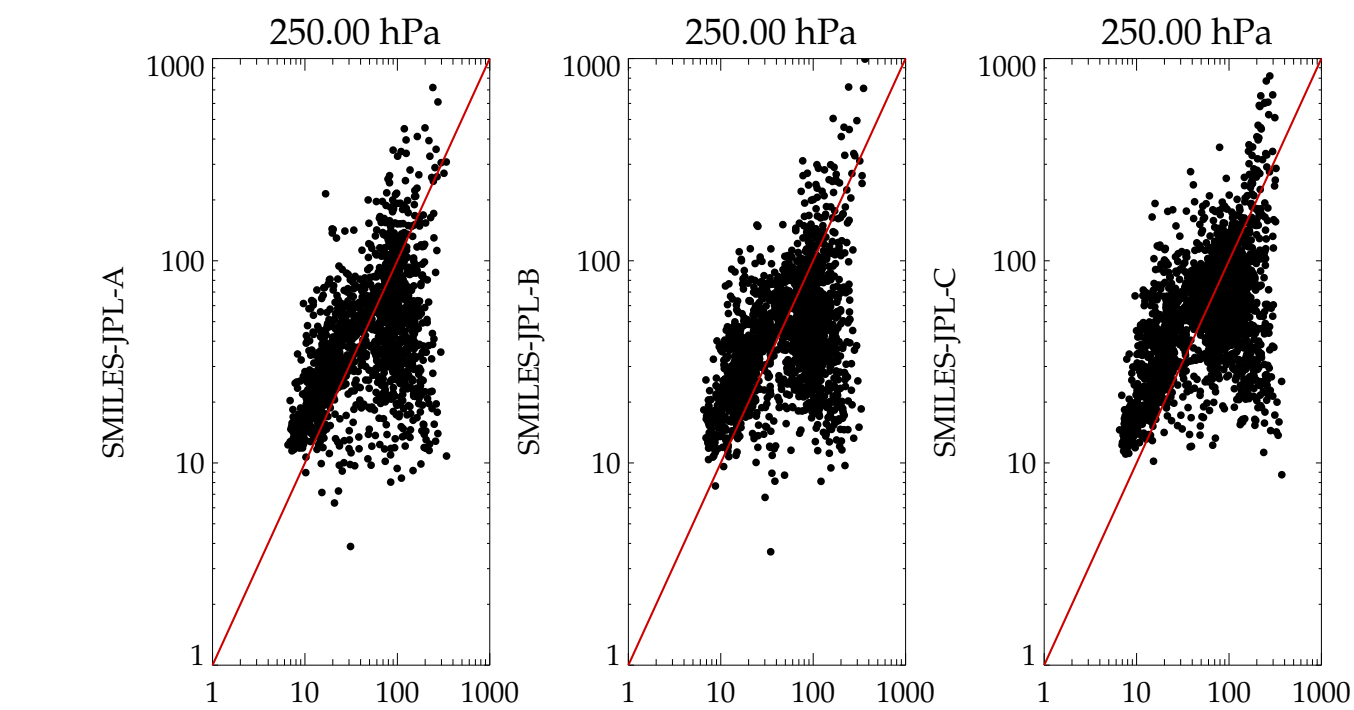

己े
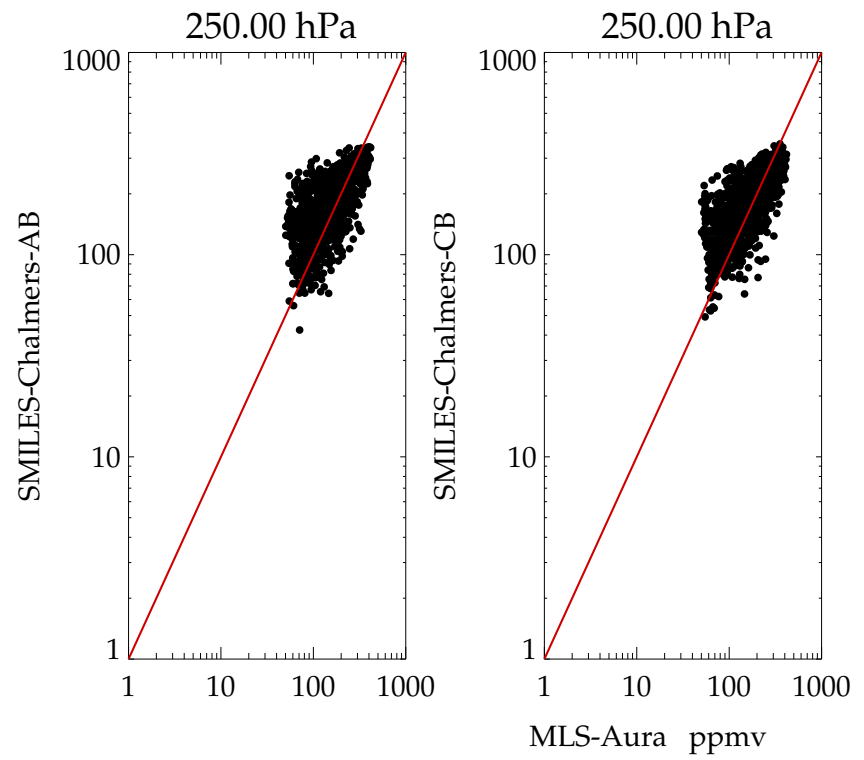

Figure S40. Scatter plot of mapped grid box values of SMILES humidity retrievals versus MLS Aura at $250 \mathrm{hPa}$. 
Mapped field grid scatter 2009.12.01-2010.03.31
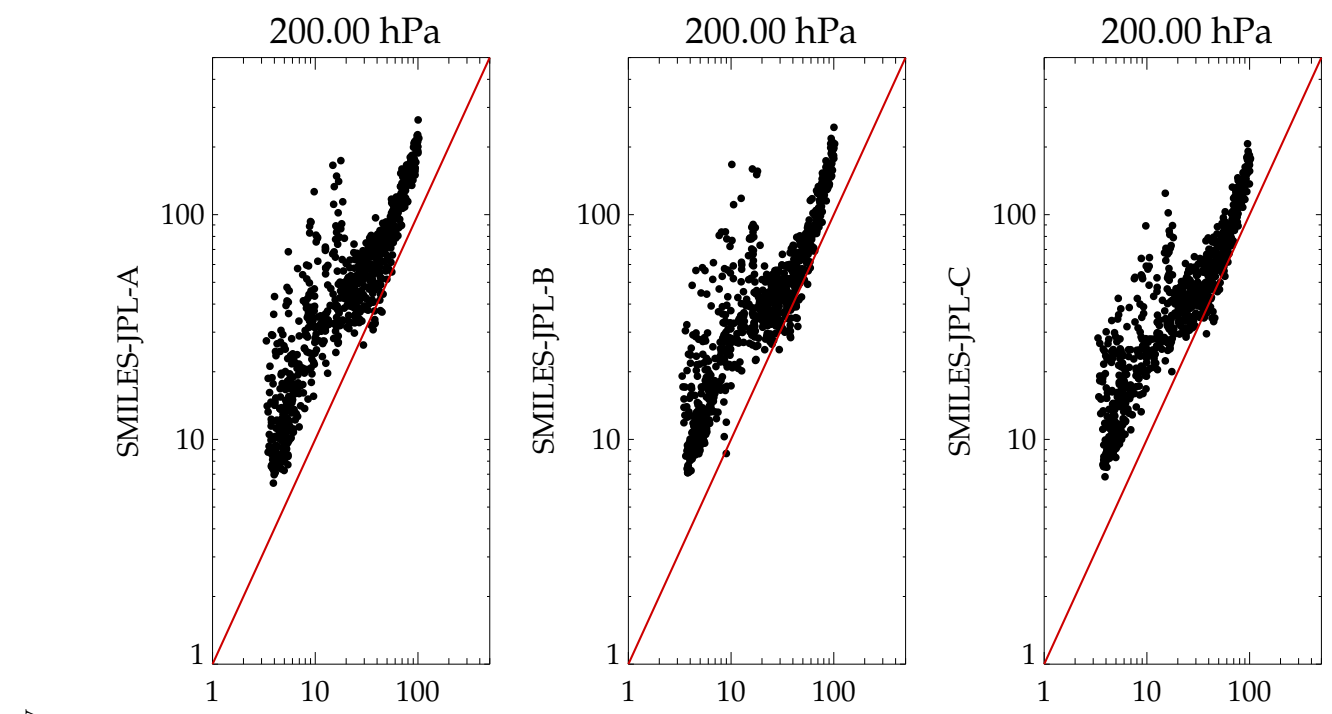

$\vec{己}$
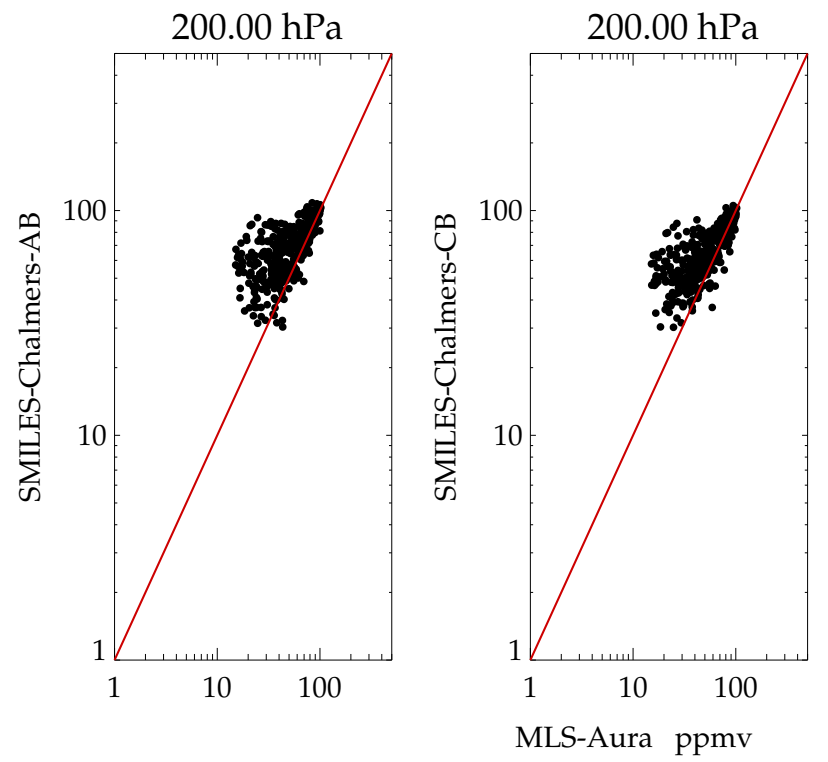

Figure S41. Scatter plot of mapped grid box values of SMILES humidity retrievals versus MLS Aura at $200 \mathrm{hPa}$. 
Mapped field grid scatter 2009.12.01-2010.03.31
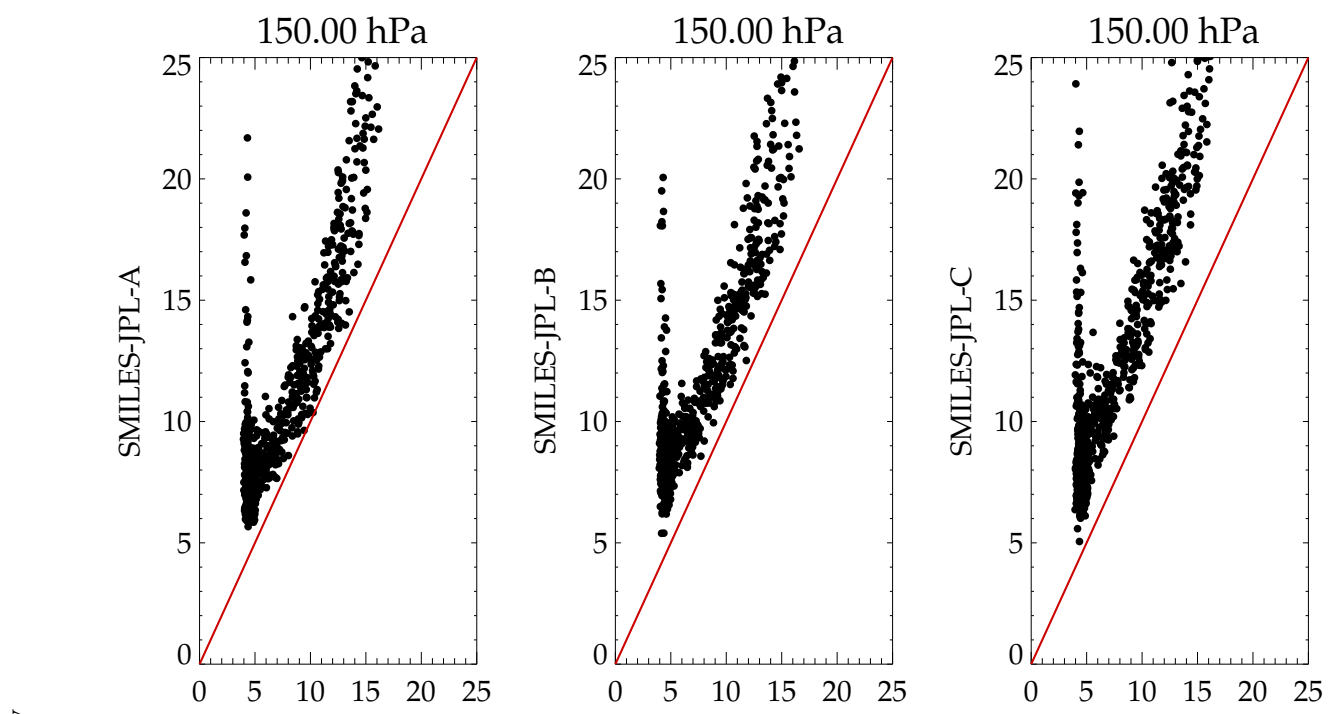

ڤ્ڤ
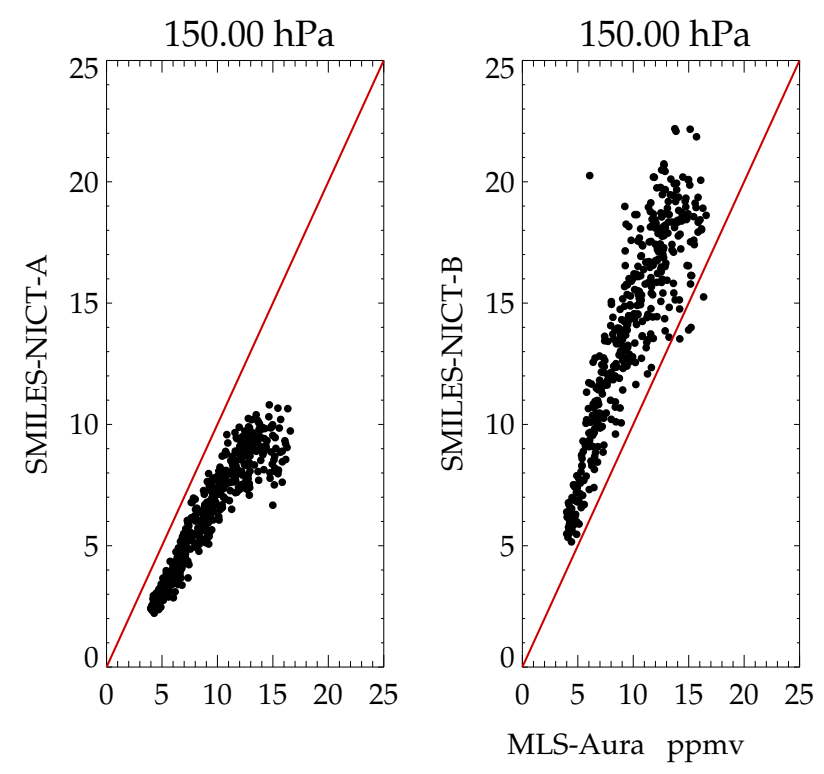

Figure S42. Scatter plot of mapped grid box values of SMILES humidity retrievals versus MLS Aura at $150 \mathrm{hPa}$. 


\subsubsection{1-2005.12.31 $\mathrm{p}=200 \mathrm{hPa}$}
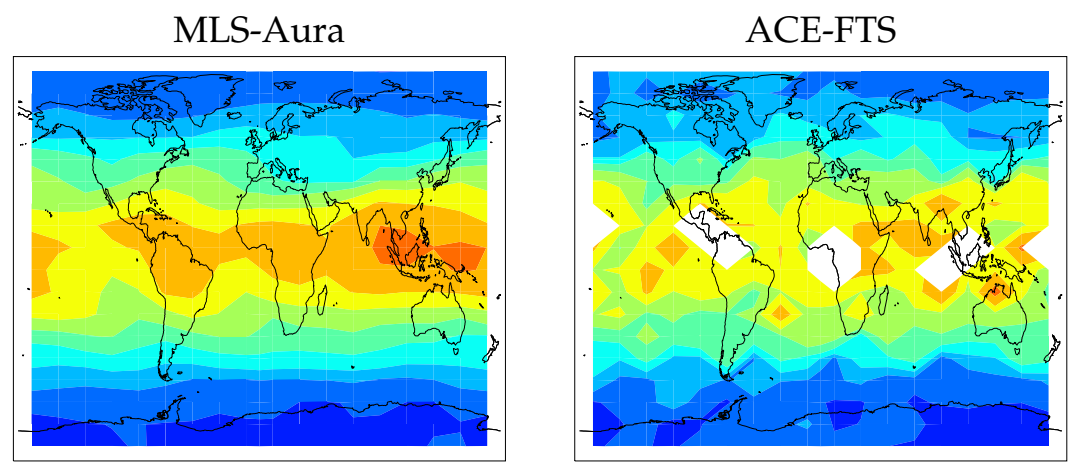

HALOE
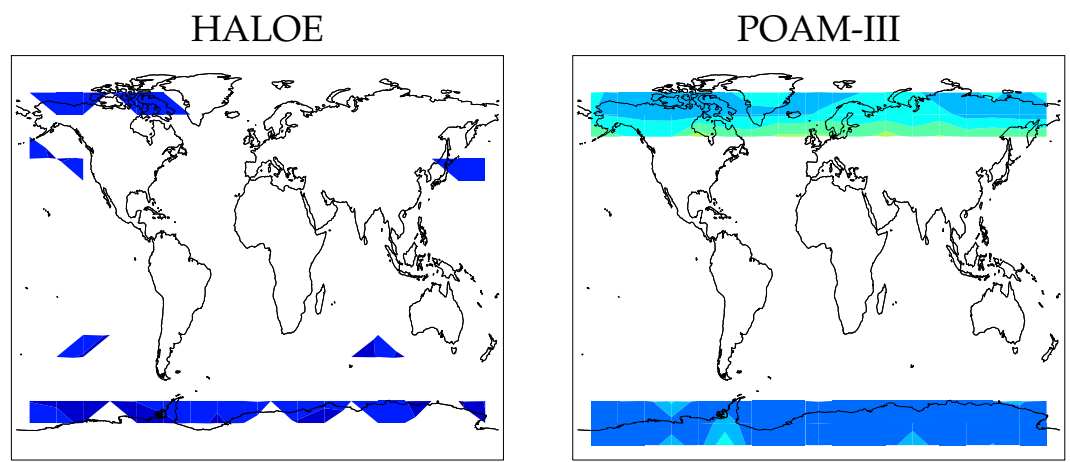

320.76

205.78

132.01

84.69

54.33

34.86

22.36 है

14.35

9.20
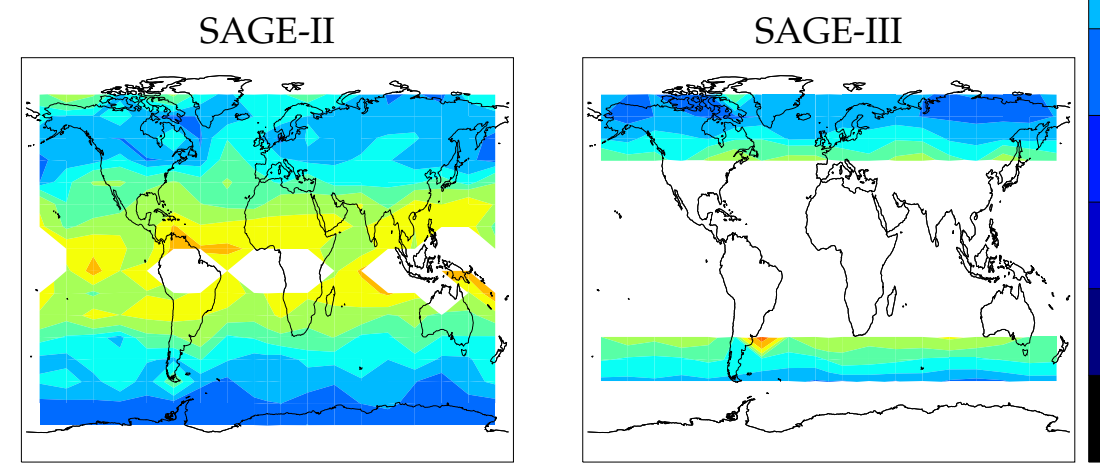

5.90

3.79

2.43

1.56

1.00

Figure S43. Gridded maps for occultation humidity retrieval products at $200 \mathrm{hPa}$. 


\subsubsection{1-2005.12.31 $\mathrm{p}=150 \mathrm{hPa}$}
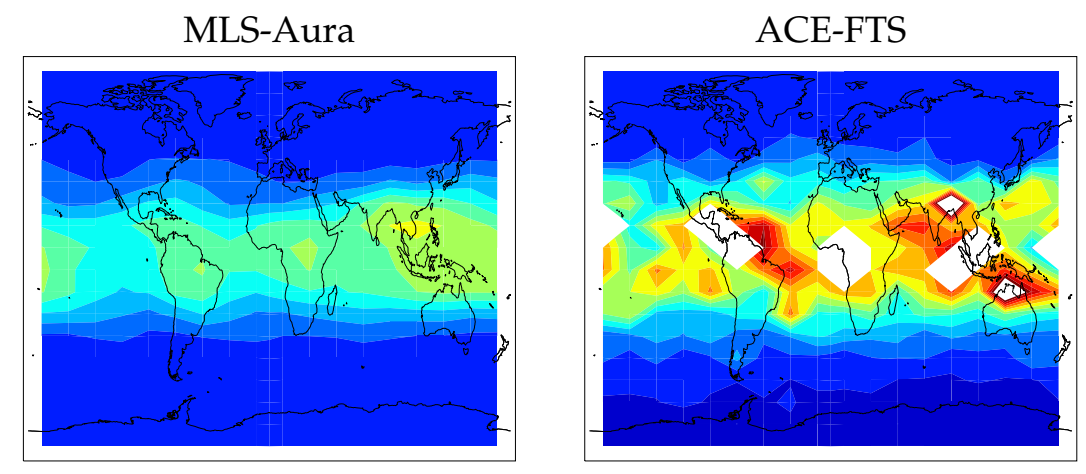

25.00

23.21

21.43

19.64
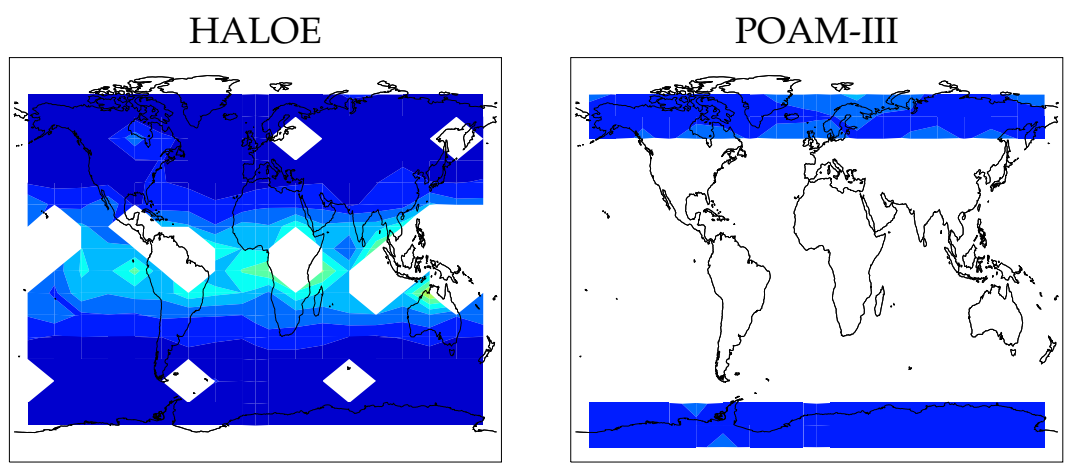

17.86

16.07

14.29

12.50 है है

10.71

8.93
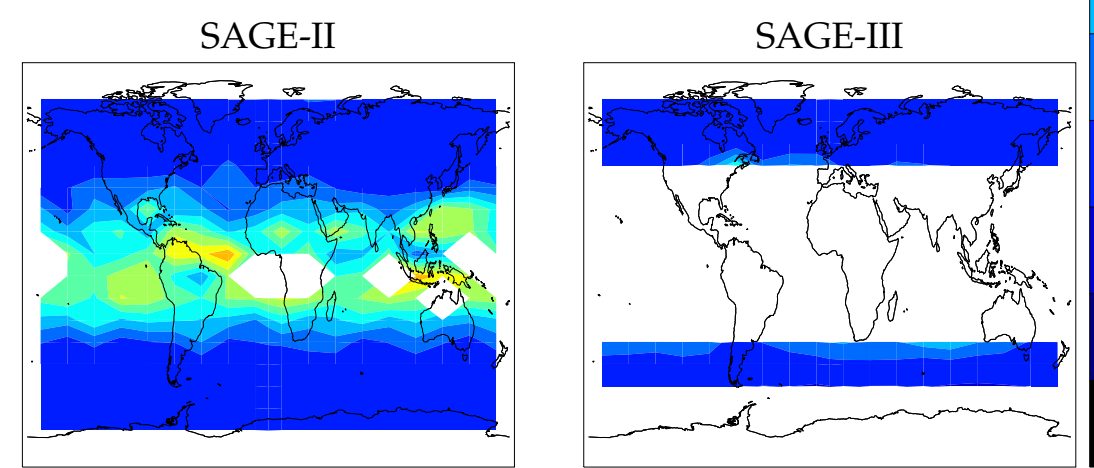

7.14

5.36

3.57

1.79

0.00

Figure S44. Gridded maps for occultation humidity retrieval products at $150 \mathrm{hPa}$. 
Mapped field grid scatter 2005.01.01-2005.12.31
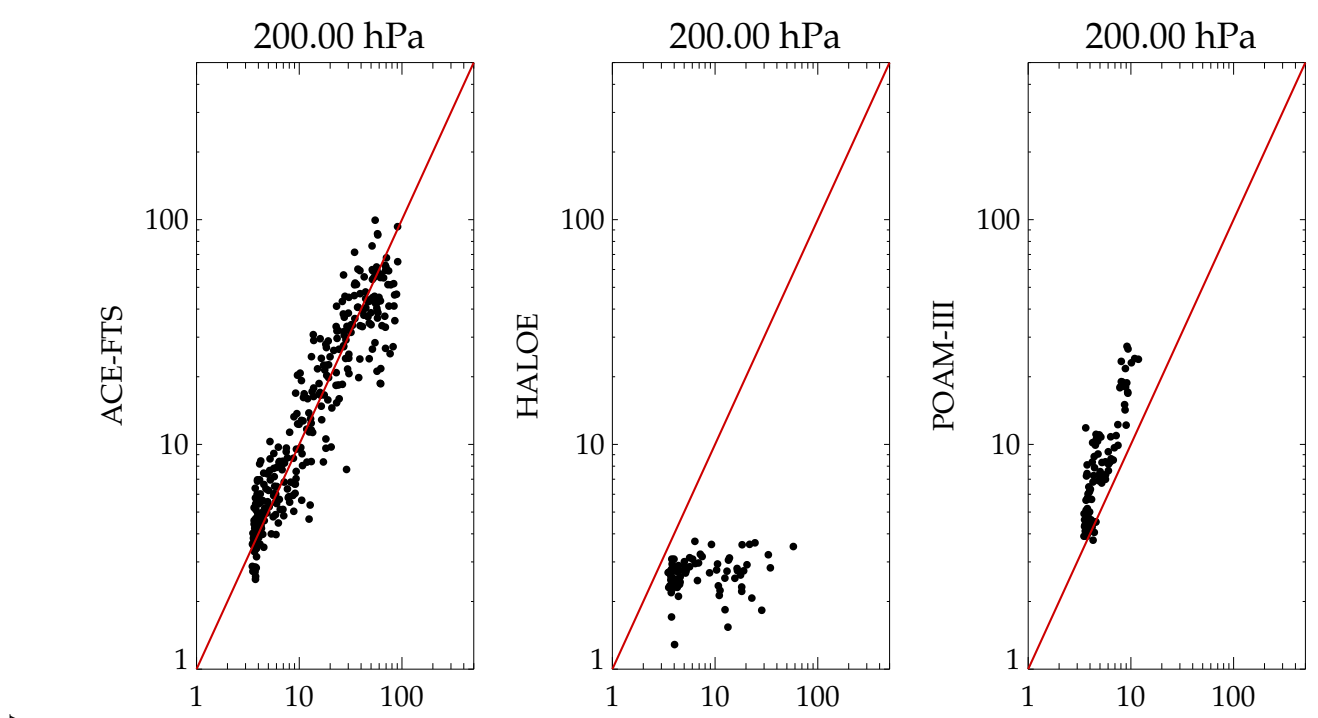

$\vec{己}$
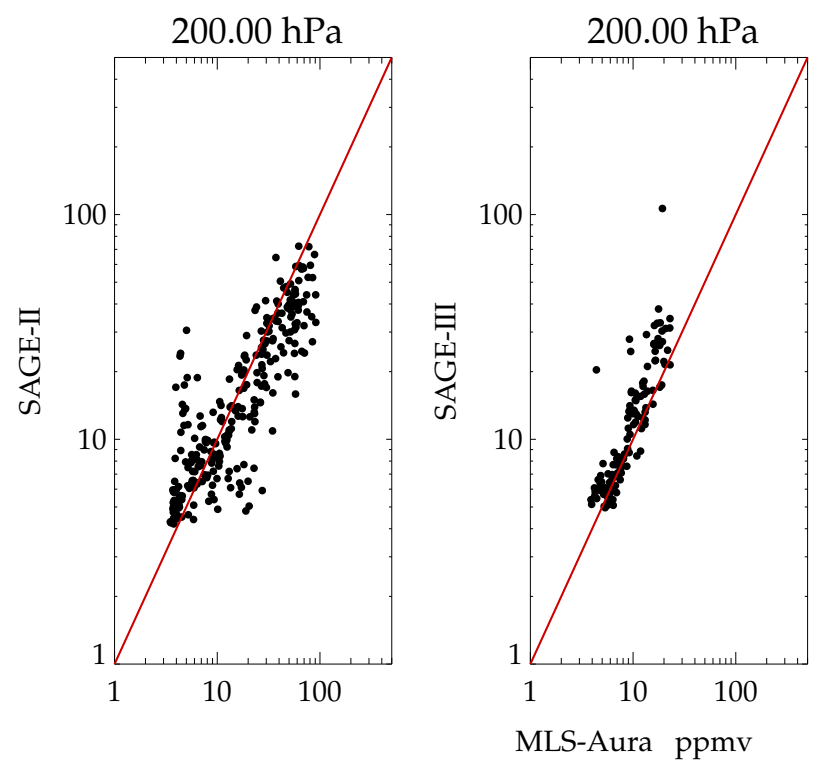

Figure S45. Scatter plot of mapped grid box values of humidity from occultation instruments versus MLS Aura at $200 \mathrm{hPa}$. 
Mapped field grid scatter 2005.01.01-2005.12.31
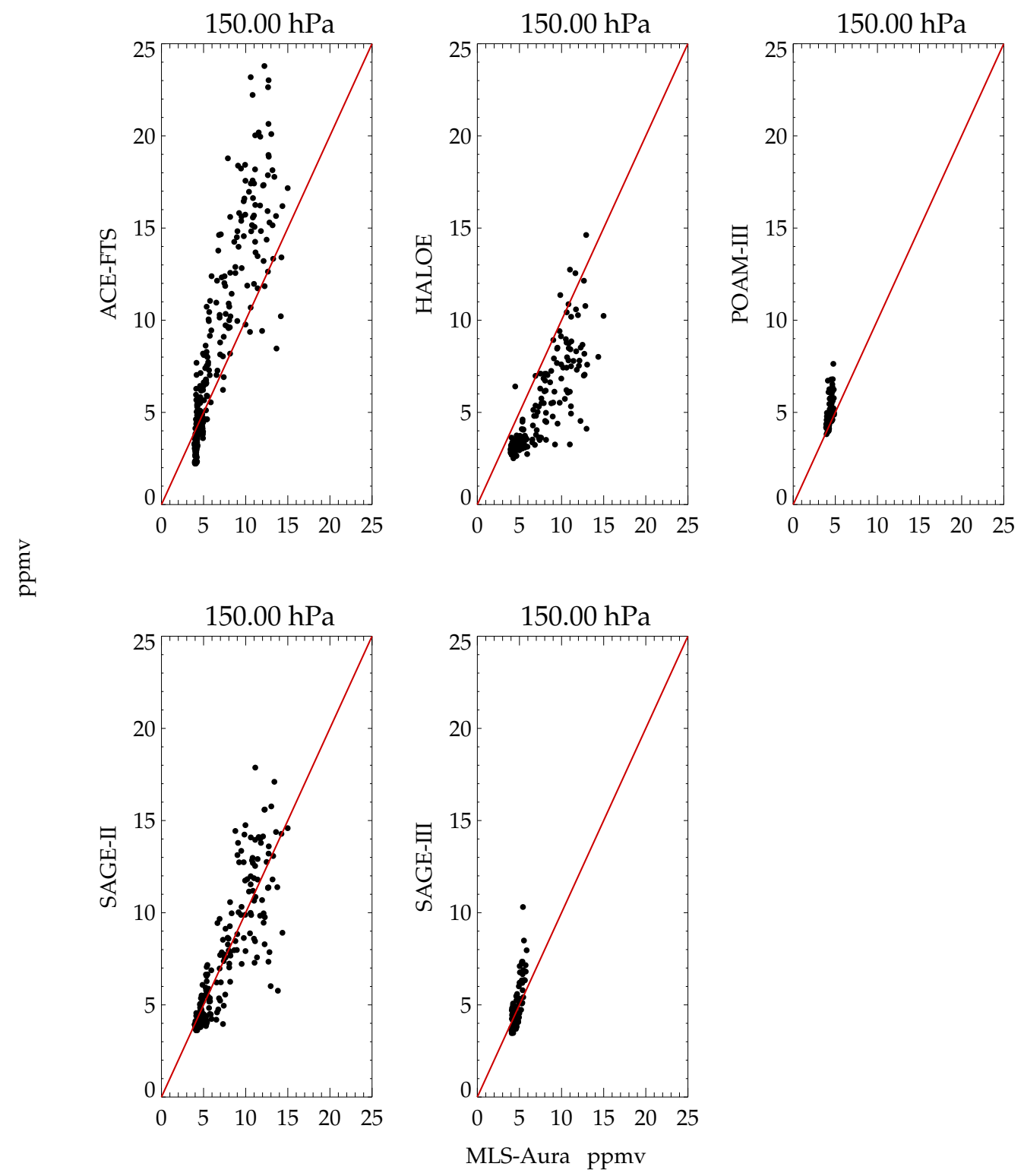

Figure S46. Scatter plot of mapped grid box values of humidity from occultation instruments versus MLS Aura at $150 \mathrm{hPa}$. 